\title{
Single-step green synthesis and characterization of gold-conjugated polyphenol nanoparticles with antioxidant and biological activities
}

This article was published in the following Dove Press journal:

International Journal of Nanomedicine

23 October 2014

Number of times this article has been viewed

\author{
Vanna Sanna ${ }^{1,2}$ \\ Nicolino Pala' \\ Giuseppina Dessì \\ Paola Manconi' \\ Alberto Mariani' \\ Sonia Dedola ${ }^{3}$ \\ Mauro Rassu ${ }^{3}$ \\ Claudia Crosio ${ }^{3}$ \\ Ciro laccarino ${ }^{3}$ \\ Mario Sechi ${ }^{1,2}$ \\ 'Department of Chemistry \\ and Pharmacy, University of \\ Sassari, Sassari, Italy; ${ }^{2}$ Laboratory \\ of Nanomedicine, Department \\ of Chemistry and Pharmacy, \\ University of Sassari, c/o Porto Conte \\ Ricerche, Tramariglio, Alghero, Italy; \\ ${ }^{3}$ Department of Biomedical Sciences, \\ University of Sassari, Sassari, Italy
}

Background: Gold nanoparticles (GNPs) are likely to provide an attractive platform for combining a variety of biophysicochemical properties into a unified nanodevice with great therapeutic potential. In this study we investigated the capabilities of three different natural polyphenols, epigallocatechin-3-gallate (EGCG), resveratrol (RSV), and fisetin (FS), to allow synergistic chemical reduction of gold salts to GNPs and stabilization in a single-step green process. Moreover, antioxidant properties of the nanosystems, as well as preliminary antiproliferative activity and apoptotic process investigation of model EGCG-GNPs on stable clones of neuroblastoma SH-SY5Y cells expressing CFP-DEVD-YFP reporter, were examined.

Methods: The GNPs were characterized by physicochemical techniques, polyphenol content, and in vitro stability. The antioxidant activity of the GNPs was also determined by 2,2-diphenyl1-picrylhydrazyl (DPPH) and 2,2'-azinobis(3-ethylbenzothiazoline-6-sulfonic acid) cation (ABTS) radical-scavenging assays. Stable clones of neuronal SH-SY5Y-CFP-DEVD-YFP were generated and characterized, and cell viability after treatment with EGCG-GNPs was assessed after 72 hours through a 3(4,5-dimethylthiazol-2yl)-5-(3-carboxymethoxyphenyl)-2(4-sulfophenyl)-2H-tetrazolium assay. Activation of the apoptotic pathways was also investigated by Western blot analysis.

Results: With a diameter in the size range of $10-25 \mathrm{~nm}$, the obtained nanoparticles (NPs) were found to contain $2.71 \%, 3.23 \%$, and $5.47 \%$ of EGCG, RSV, and FS, respectively. Nanoprototypes exhibited remarkable in vitro stability in various media, suggesting that NP surface coating with phytochemicals prevents aggregation in different simulated physiological conditions. The scavenging activities for DPPH and ABTS were highly correlated with EGCG, RSV, and FS content. Moreover, high correlation coefficients between the ABTS and DPPH values were found for the prepared nanosystems. EGCG-GNPs induce a dose-dependent reduction on SH-SY5YCFP-DEVD-YFP cell viability that is likely to involve the activation of the apoptotic pathways, similarly to free EGCG, as suggested by the processing of the CFP-DEVD-YFP reporter.

Conclusion: These results prompted us to propose the ecofriendly synthesized EGCG-, RSV-, and FS-based nanogold conjugates as suitable carriers for bioactive polyphenols to be used for the treatment of disorders associated with oxidative stress, including neurodegenerative disorders, cardiovascular disease, and cancer.

Keywords: gold nanoparticles, epigallocatechin-3-gallate, resveratrol, fisetin, antioxidant activity, SH-SY5Y-CFP-DEVD-YFP cells

\section{Introduction}

In recent years, the synthesis and biofunctionalization of water-dispersible gold nanoparticles (GNPs) have attracted intense interest due to their potential use in biology and medicine. ${ }^{1-4}$ Some of the major biomedical applications of GNPs include gene 
therapy, ${ }^{5}$ protein delivery, ${ }^{6}$ cancer diagnosis,,${ }^{7,8}$ photothermal and photodynamic therapy, ${ }^{1}$ and delivery of antitumor

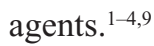

The most commonly used method for the synthesis of GNPs is the reduction of chloroauric acid by trisodium citrate. ${ }^{10}$ Other chemical and physical techniques involve the use of powerful and highly toxic reducing agents such as hydrazine and sodium borohydride, as well as high temperature and pressure conditions. ${ }^{11}$ Moreover, the synthesis of the GNPs can be performed in the presence of a stabilizer in order to modify the surface by physisorption, specific recognition, and electrostatic interactions to ensure stability, ${ }^{12,13}$ which is an important determinant for the potential use of gold nanoconjugated as therapeutic agents. ${ }^{14}$ In order to minimize or eliminate further chemical interventions and to improve the sustainability of the process, alternative biosynthetic green methods that utilize plant-based phytochemicals for reduction of metal ions provide an inherently green approach to nanotechnology, also referred to as "green nanotechnology". ${ }^{15}$

Recently, several studies have demonstrated the dual role of whole plant extracts and pure compounds isolated from plants as effective reducing agents and as stabilizers, to provide a robust coating on the biocompatible GNPs. ${ }^{16-20}$ The reactive phytochemical species include polyphenols such as flavonoids and nonflavonoids, the most abundant antioxidants in human diets, usually found in some fruits, vegetables, and beverages. ${ }^{21,22}$

In this study we investigated the capability of three different natural polyphenols, epigallocatechin-3-gallate (EGCG), resveratrol (RSV), and fisetin (FS), to provide synergistic chemical reduction of gold salts to GNPs, as well as stabilization in a single-step green process (Figure 1).

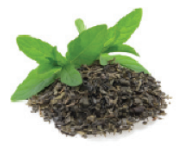

$\sqrt{7}$<smiles>COc1cc(O)cc(O)c1CC(OC(=O)c1cc(O)c(O)c(O)c1)c1cc(O)c(O)c(O)c1</smiles>

(-)-Epigallocatechin 3-gallate

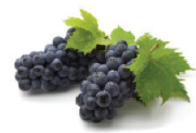

$\sqrt{3}$<smiles>Oc1ccc(/C=C/c2cc(O)cc(O)c2)cc1</smiles>

Resveratrol

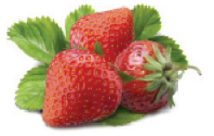

$\sqrt{7}$

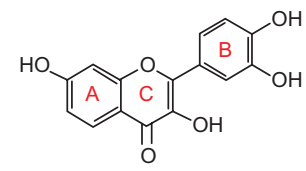

Fisetin

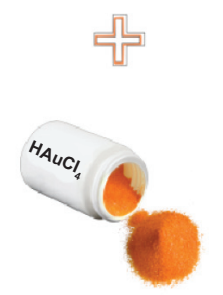

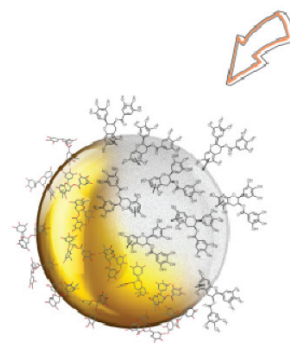

EGCG-GNPs

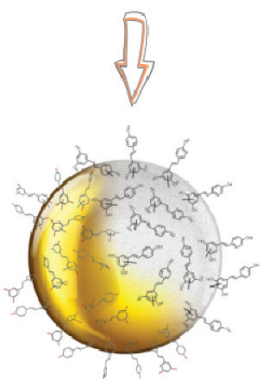

RSV-GNPs

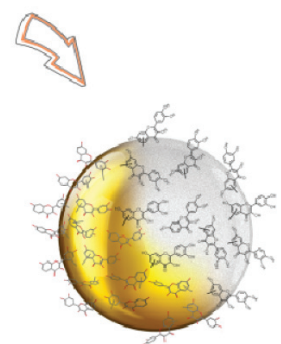

FS-GNPs

Figure I The work plan: from polyphenols to nanogolds. Chemical structures of EGCG, RSV, and FS are included. Abbreviations: EGCG, epigallocatechin-3-gallate; RSV, resveratrol; FS, fisetin; GNPs, gold nanoparticles. 
These polyphenols, with different chemical structures, have been selected because they have received a great deal of interest worldwide as potent bioactive substances endowed with chemopreventive/therapeutic activities because of their anti-invasive and antiproliferative effects in a wide variety of tumor cells, ${ }^{23-26}$ with an increased effectiveness when formulated into a variety of nanosystems. ${ }^{27-31}$

On the other hand, the functionalization of GNPs' surface with these bioactive substances might provide a new useful approach as delivery and therapeutic tools, in particular by improving the biopharmaceutical properties of these polyphenols, such as their poor bioavailability and stability, as well as their resistance to specific/nonspecific metabolic processes.

As far as the chemical structure is concerned, EGCG (ie, [-]-epigallocatechin-3-gallate) is a flavonoid constituted by a dihydroxybenzopyran scaffold carrying a pyrogalloltype structure on the B-ring and a galloyl moiety in the D-ring (Figure 1). RSV (ie, trans-3,5,4'-trihydroxystilbene) is a triphenolic phytoalexin having the trans-stilbene skeleton, while FS (ie, 3, 3', $4^{\prime}, 7$-tetrahydroxyflavone) is a flavone-3-ol that bears a catecholic structural unit in the B-ring and the two hydroxyl groups located in the C-and A-rings (Figure 1).

To date, few examples of EGCG-conjugated nanogold have been reported. In a pioneering work, Nune et $\mathrm{al}^{32}$ investigated the role of phytochemicals present in black tea leaves and commercially available catechins to provide GNPs with therapeutic potential. More recently, they demonstrated that biocompatible radioactive GNPs stabilized by EGCG bind with excellent affinity to laminin receptors overexpressed in prostate tumor cells, and reduce prostate tumor volumes in vivo. ${ }^{33}$ Furthermore, Hsieh et $\mathrm{al}^{34}$ reported on the synthesis, characterization, antioxidant activity, cytotoxicity in vitro, as well as anticancer activity in vivo of EGCG-conjugated nanogold.

The reaction mechanism for the formation of GNPs in aqueous solution by polyphenols reduction has been proposed in several reports. ${ }^{35-37}$ Polyphenols such as EGCG demonstrated to be efficient ligands for metal ions and multiple phenolic hydroxyls are able to bind $\mathrm{Au}^{3+}$ ions by forming stable chelating rings. As a consequence of complexation, a redox reaction occurs, and the chelated $\mathrm{Au}^{3+}$ ions are reduced to $\mathrm{Au}^{0}$ atoms in situ, while a part of the phenolic hydroxyls is simultaneously oxidized to corresponding quinone. Thus, polyphenolic molecules result in being physically attached onto the surface of nanogold particles, and the binding of an $\mathrm{O}$ atom of the polyphenol molecule to the GNPs' surfaces is presumably due to the electron transfer from $\mathrm{O}$ atoms to $\mathrm{Au}^{3+}$ ions, as confirmed by computational studies. ${ }^{38} \mathrm{On}$ the other hand, the high density of free hydroxyl groups, especially in EGCG-GNPs, could lead to extensive inter- and intramolecular hydrogen bonding, which favors formation of unique supramolecular assemblies.

However, so far, the potential of RSV and FS as reducing agents for the synthesis of GNPs, as well as on their putative effectiveness when attached on the GNPs' surface, has not been explored yet. Therefore, it could also be interesting to preliminarily explore the behaviour of GNPs decorated with these polyphenols (ie, EGCG, RSV, and FS).

More specifically, we sought to test whether or not the intrinsic antioxidant activity displayed by the free compounds was retained when they are adsorbed to the nanoparticles (NPs) surface. Moreover, we wanted to investigate the ability of these engineered capped GNPs in regulating and modulating cellular responses, by evaluating their antiproliferative activity and induction of apoptosis on stable clones of neuroblastoma SH-SY5Y cells expressing CFP-DEVD-YFP reporter.

Herein, we report on "green nanosynthesis" and characterization, in terms of physicochemical properties and polyphenols content, of novel prototypes of polyphenolcapped GNPs (Figure 1) suitable for biomedical finalization. In vitro stability of the colloidal suspensions in various physiological media was also investigated. Furthermore, the capability of obtained GNPs for scavenging free radicals was demonstrated in vitro by following the reaction with stable 2,2-diphenyl-1-picrylhydrazyl (DPPH) and 2,2'-azinobis (3-ethylbenzothiazoline-6-sulfonic acid) cation (ABTS) radicals. Finally, cell viability after treatment with an EGCG-GNP model was evaluated through a 3(4,5-dimethylthiazol-2yl)-5(3-carboxymethoxyphenyl)-2-(4-sulfophenyl)-2H-tetrazolium (MTS) assay, and activation of the apoptotic pathways was investigated by Western blot analysis.

\section{Materials and methods Chemicals and reagents}

Tetrachloroauric acid (III) trihydrate $\left(\mathrm{HAuCl}_{4} \cdot 3 \mathrm{H}_{2} \mathrm{O}\right.$, $>99.5 \%$ purity), DPPH, ABTS, and GNPs (15 nm diameter, stabilized suspension in citrate buffer) were purchased from Sigma-Aldrich (Steinheim, Germany). EGCG ( $\geq 99 \%$ purity) and RSV ( $\geq 98 \%$ purity) were purchased from Zhejiang Yixin Pharmaceutical Co., Ltd. (Zhejiang, People's Republic of China). FS ( $>98 \%$ purity) was purchased from Shaanxi Taiji Huaqing Technology Co., Ltd. (Shaanxi, People's Republic of China).

\section{Synthesis of GNPs}

The synthesis of GNPs was carried out by modification of a method previously reported. ${ }^{32}$ Aqueous solution of 
$\mathrm{HAuCl}_{4} \cdot 3 \mathrm{H}_{2} \mathrm{O}(10 \mathrm{mM}, 1.0 \mathrm{~mL})$ and polyphenol $(\mathrm{EGCG}$, $\mathrm{RSV}$, or FS) solution (1.0 mM, 5.0 mL) was mixed under gentle stirring at room temperature. EGCG was solubilized in water, while RSV and FS were solubilized in ethanol:water mixture $(1: 4, \mathrm{v} / \mathrm{v})$. The color of the mixture containing EGCG and RSV turned purple-red from pale yellow within a few minutes, indicating the formation of GNPs. For the solution containing FS the color appeared orange then yellow and purple-red after about 10 minutes. The reaction mixture was stirred for an additional 30 minutes. The GNPs were collected by centrifugation $\left(20,000 \mathrm{rpm}, 10\right.$ minutes, $\left.15^{\circ} \mathrm{C}\right)$ and washed three times with water to ensure that no unbound EGCG, $\mathrm{RSV}$, or FS molecules remained in the NP dispersion.

\section{Characterization}

The ultraviolet-visible (UV-vis) absorbance spectrum of diluted aqueous NP suspensions was recorded using a Cary 3 UV-vis spectrophotometer (Varian Medical Systems, Inc., Palo Alto, CA, USA).

The morphology and size of the GNPs were characterized by transmission electron microscopy (TEM) (FEI Tecnai G12; FEI, Hillsboro, OR, USA). A drop of sample was placed on a formvar-coated 400 mesh copper grid, followed by drying the sample at ambient conditions before it was attached to the sample holder on the microscope.

The phase characteristics of the as-prepared products were examined by powder X-ray diffraction (XRD; Bruker D8 Advance diffractometer) using $\mathrm{Cu} \mathrm{K} \alpha$ radiation $(\lambda=1.54056 \AA)$, operating at $40 \mathrm{kV}$ and $40 \mathrm{~mA}$. Results were recorded in the $2 \theta$ range from $20^{\circ}$ to $80^{\circ}$ with a scanning step of $0.02^{\circ}$ per step and 2 seconds per point.

Infrared spectra were collected on a Fourier transform infrared (FT-IR) spectrometer (Bruker Optics, Ettlingen, Germany) at a resolution of $4 \mathrm{~cm}^{-1}$ in $\mathrm{KBr}$ pellets. The measurements were performed on EGCG, RSV, and FS raw materials as well as on GNPs produced.

\section{Determination of polyphenol content}

The polyphenol content was determined by spectrophotometry, using EGCG, RSV, and FS as standard, with some modifications of the method described..$^{39,40}$ Briefly, $0.1 \mathrm{mg}$ of the GNPs was transferred to separate tubes containing $0.5 \mathrm{~mL}$ of a 1/10 dilution of Folin-Ciocalteu's reagent in water. Then, $1.0 \mathrm{~mL}$ of a sodium carbonate solution $(7.5 \% \mathrm{w} / \mathrm{v})$ was added. The tubes were then allowed to stand at $30^{\circ} \mathrm{C}$ for 30 minutes before absorbance at $760 \mathrm{~nm}$ was measured. The polyphenol content was expressed as EGCG, RSV, and FS equivalents in $\mu \mathrm{g} / 100 \mu \mathrm{g}$ GNPs. The concentration of polyphenol in samples was derived from the standard curve of EGCG, RSV, and FS raw materials ranging from 0.1 to $10 \mu \mathrm{g} / \mathrm{mL}$ (EGCG: $\mathrm{y}=0.0563 \mathrm{x}+0.0101 ; R^{2}=0.9993 ; \mathrm{RSV}: \mathrm{y}=0.0765 \mathrm{x}+0.0086$; $\left.R^{2}=0.9990 ; \mathrm{FS}: \mathrm{y}=0.0649 \mathrm{x}+0.0104 ; R^{2}=0.9991\right)($ Figure $\mathrm{S} 1)$.

\section{In vitro stability}

In vitro stability studies of GNPs were performed in different media as previously reported. ${ }^{32}$ Typically, colloidal NP solution was added to glass vials containing $1.5 \mathrm{~mL}$ of $0.5 \%$ bovine serum albumin (BSA), 0.5\% cysteine, 5.0\% sodium chloride $(\mathrm{NaCl})$, and phosphate-buffered solution (PBS) $\mathrm{pH} 5, \mathrm{pH} 7$, and pH 9, respectively, and incubated for 3 hours at room temperature. The stability of GNPs was measured by monitoring UV absorbance at 30-minute intervals. A negligible change in UV-vis plasmon band confirmed the retention of nanoparticulate composition in all mixtures.

\section{Antioxidant test: DPPH assay}

The capacity of plant materials to catalyze the decomposition of free radicals can be determined by their ability to reduce the concentration of the stable free radical DPPH. ${ }^{41}$ The method previously described by Gülçin ${ }^{42}$ was used with slight modifications. Stock solutions of DPPH were prepared at $0.1 \mathrm{mmol} / \mathrm{L}$ in ethanol and stored at $-20^{\circ} \mathrm{C}$ until needed. An appropriate amount of GNPs dispersed in ethanol $(500 \mu \mathrm{L})$ was added to $1.0 \mathrm{~mL}$ DPPH solution in a test tube to give different final concentrations of NPs $(25,50,100$, and $200 \mu \mathrm{g} / \mathrm{mL}$ ). The mixture was sonicated for 5 minutes and then left to stand in the dark. The supernatant containing only DPPH was obtained by centrifuging at 20,000 rpm for 5 minutes. Nonfunctionalized GNPs commercially available were tested as comparison at the same concentration.

The absorption of the supernatant was measured at $517 \mathrm{~nm}$ against blank samples lacking scavenger using a UV-vis spectrophotometer. The decrease in absorbance was determined at 5-minute intervals up to 30 minutes. The scavenging percentage was calculated using the following formula:

$$
\begin{aligned}
& \text { DPPH-scavenging } \\
& \text { effect }(\%)
\end{aligned}=\left[\frac{\left(\mathrm{ABS}_{\text {Control }}-\mathrm{ABS}_{\text {Sample }}\right)}{\mathrm{ABS}_{\text {Control }}}\right] \times 100
$$

where $\mathrm{ABS}_{\text {Control }}$ and $\mathrm{ABS}_{\text {Sample }}$ are absorption of control DPPH and sample applied DPPH at $517 \mathrm{~nm}$, respectively.

\section{Antioxidant test: ABTS assay}

The ABTS radical cation decolorization test is a spectrophotometric method widely used for the assessment of antioxidant activity of various substances. The experiment was carried out by adapting a method previously reported. ${ }^{43}$ 
In brief, a mixture containing $99 \mathrm{~mL}$ of ABTS solution $\left(5.0 \times 10^{-4} \mathrm{M}\right)$ and $1.0 \mathrm{~mL}$ of sodium persulfate $\left(6.89 \times 10^{-3} \mathrm{M}\right)$ was stored in the dark at room temperature for 16 hours before use. The produced ABTS •+ solution was diluted with ethanol to obtain an absorbance of approximately 0.85 at $734 \mathrm{~nm}$. After the addition of $900 \mu \mathrm{L}$ of diluted ABTS solution to $100 \mu \mathrm{L}$ of sample, the absorbance reading was taken at 1-minute intervals up to 5 minutes. Nonfunctionalized GNPs commercially available were used as comparison. A sample blank was run in each assay. All determinations were carried out in triplicate after centrifugation at 20,000 rpm for 1 minute. The percentage radical-scavenging activity was calculated as follows:

$$
\begin{aligned}
& \text { ABTS } \bullet+- \\
& \text { scavenging effect }(\%)
\end{aligned}=\left[\frac{\left(\mathrm{ABS}_{\text {Control }}-\mathrm{ABS}_{\text {Sample }}\right)}{\mathrm{ABS}_{\text {Control }}}\right] \times 100
$$

where $\mathrm{ABS}_{\text {Control }}$ is absorbance of a control lacking any radical scavenger and $\mathrm{ABS}_{\text {Sample }}$ is absorbance of the remaining ABTS $\bullet+$ in the presence of a scavenger. ${ }^{44}$

\section{Cell culture}

Human neuroblastoma SH-SY5Y cells (American Type Culture Collection number CRL-2266) were cultured in Dulbecco's Modified Eagle Medium F-12 (DMEM/F12) ground (Life Technologies, Carlsbad, CA, USA) in the presence of $10 \%$ fetal calf serum (Life Technologies) inactivated at $56^{\circ} \mathrm{C}$ for 30 minutes. The cells are grown in an incubator at $37^{\circ} \mathrm{C}$ in a humidified atmosphere containing $5 \%$ $\mathrm{CO}_{2}$. Stable clones expressing CFP-DEVD-YFP (Addgene company code 24537 ) were obtained by transfecting cells with LipofectAMINE LTX and PLUS reagent (Life Technologies) using $1.5 \mu \mathrm{g} \mathrm{DNA} / 5-7 \times 10^{5}$ cells according to the manufacturer's protocol. The different SH-SY5Y clones were maintained under selection by $400 \mu \mathrm{g} / \mathrm{mL}$ of $\mathrm{G} 418$. Individual clones expressing antibiotic resistance were picked after 14 days of selection, moved into a 48 -well plate, and maintained in selective medium until confluence growth. Different individual clones were analyzed for CFP-DEVDYFP expression upon treatment with the caspase-3 activator staurosporine $(1 \mu \mathrm{g} / \mathrm{mL})$.

\section{Assessment of cell viability}

The viability of control SH-SY5Y-CFP-DEVD-YFP cells was calculated after 72 hours through an MTS assay. Cell viability was assessed by a colorimetric assay using the MTS assay (CellTiter 96 Aqueous One Solution Assay; Promega Corporation, Madison, WI, USA), according to the manufacturer's instructions. Absorbance at $490 \mathrm{~nm}$ was measured in a multilabel counter (Victor X5; Perkin Elmer, Waltham, MA, USA).

\section{Immunofluorescence}

One $\times 10^{5}$ cells were grown on a cover-glass for 24 hours and treated with staurosporine $(1.0 \mu \mathrm{M})$ for 2 hours. Cells were washed twice with PBS $1 \mathrm{X}$ and then fixed with $1 \mathrm{~mL}$ of $4 \%$ paraformaldehyde/PBS 1X for 10 minutes. Before analysis, cells were mounted using Mowiol mounting medium ${ }^{45}$ and fluorescence was revealed with a Leica TCS SP5 confocal microscope with LAS lite 170 image software.

\section{Western blot analysis}

Western blot analysis was performed as previously described. ${ }^{46}$ Briefly, protein content was determined using the Bradford protein assay. Equal amounts of protein extracts were resolved by standard sodium dodecyl sulfate-polyacrylamide gel electrophoresis. Samples were electroblotted onto Protan nitrocellulose (Schleicher and Schuell BioScience GmbH, Dassel, Germany). Membranes were incubated with $3 \%$ low-fat milk in $1 \mathrm{X}$ PBS-Tween $0.05 \%$ solution with rabbit anti-green fluorescent protein (anti-GFP; Enzo Life Science, Farmingdale, NY, USA; $1: 1,000$ final dilution) or mouse anti- $\beta$-actin (Sigma-Aldrich; $1: 1,000$ final dilution) for 16 hours at $4^{\circ} \mathrm{C}$. Goat antimouse immunoglobulin $\mathrm{G}(\mathrm{IgG})$ peroxidase-conjugated antibody (Millipore Corporation, Billerica, MA, USA; 1:2,500 final dilution) or goat antirabbit IgG peroxidase-conjugated antibody (Millipore; 1:2,500 final dilution) were used to reveal immunocomplexes by enhanced chemiluminescence (Pierce Biotechnology, Rockford, IL, USA).

\section{Statistical analysis}

Data are expressed as the mean \pm standard deviation of experiments carried out in triplicate. The significance of differences was assessed by one-way analysis of variance. Individual differences were evaluated using a nonparametric post hoc test (Tukey's test) and considered statistically significant at $P<0.05$.

\section{Results and discussion Synthesis and characterization of GNPs}

In this study, green reduction of $\mathrm{Au}^{3+}$ to GNPs by bioactive polyphenols EGCG, RSV, and FS was performed by simply mixing polyphenol and metal gold salt solutions at room temperature. The reaction was complete within minutes and the formation of EGCG-, RSV-, and FS-capped GNPs could be followed by color change from yellow to reddish violet 


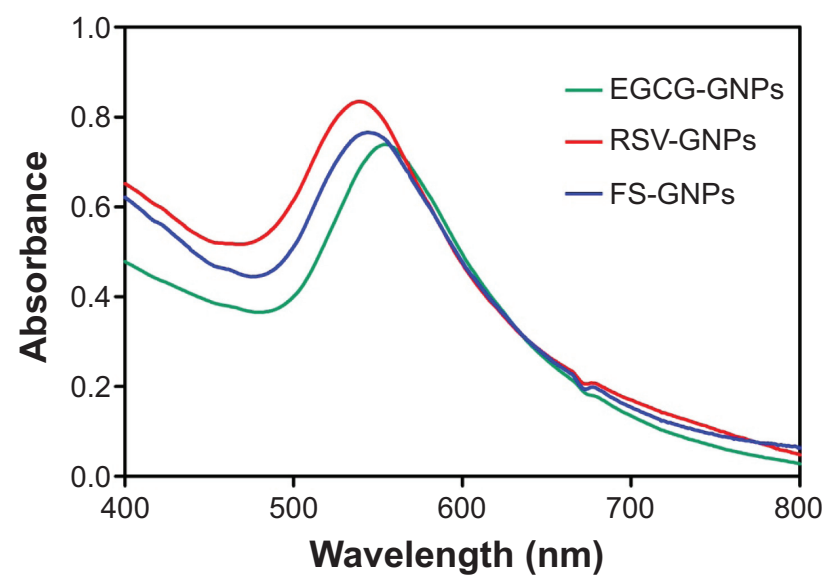

Figure 2 Ultraviolet-visible spectra of stable GNPs synthesized by EGCG, RSV, and FS polyphenols.

Notes: Wavelength expressed in nm. EGCG-GNPs, RSV-GNPs, and FS-GNPs indicate polyphenol-capped nanogold.

Abbreviations: EGCG, epigallocatechin-3-gallate; RSV, resveratrol; FS, fisetin; GNPs, gold nanoparticles.
(Figure S1), due to excitation of surface plasmon resonance (SPR) vibration of GNPs.

In UV-vis absorbance spectra (Figure 2), synthesis of GNPs was confirmed by characteristic SPR peak centered at 554, 540, and $544 \mathrm{~nm}$ for EGCG-, RSV-, and FS-capped GNPs, respectively. The significant SPR shift to higher wavelengths observed for EGCG-GNPs suggests an increase in particle size compared with NP batches prepared using RSV and FS. ${ }^{47}$

The size and shape of the GNPs were investigated with TEM. The images reported in Figure 3 show that EGCGGNPs (Figure 3A) are in variable shapes. Most of them are spherical in nature, followed by occasionally triangular and rarely hexagonal.

On the other hand, a more uniform size distribution and homogeneous spherical shape of RSV and FS-GNPs were
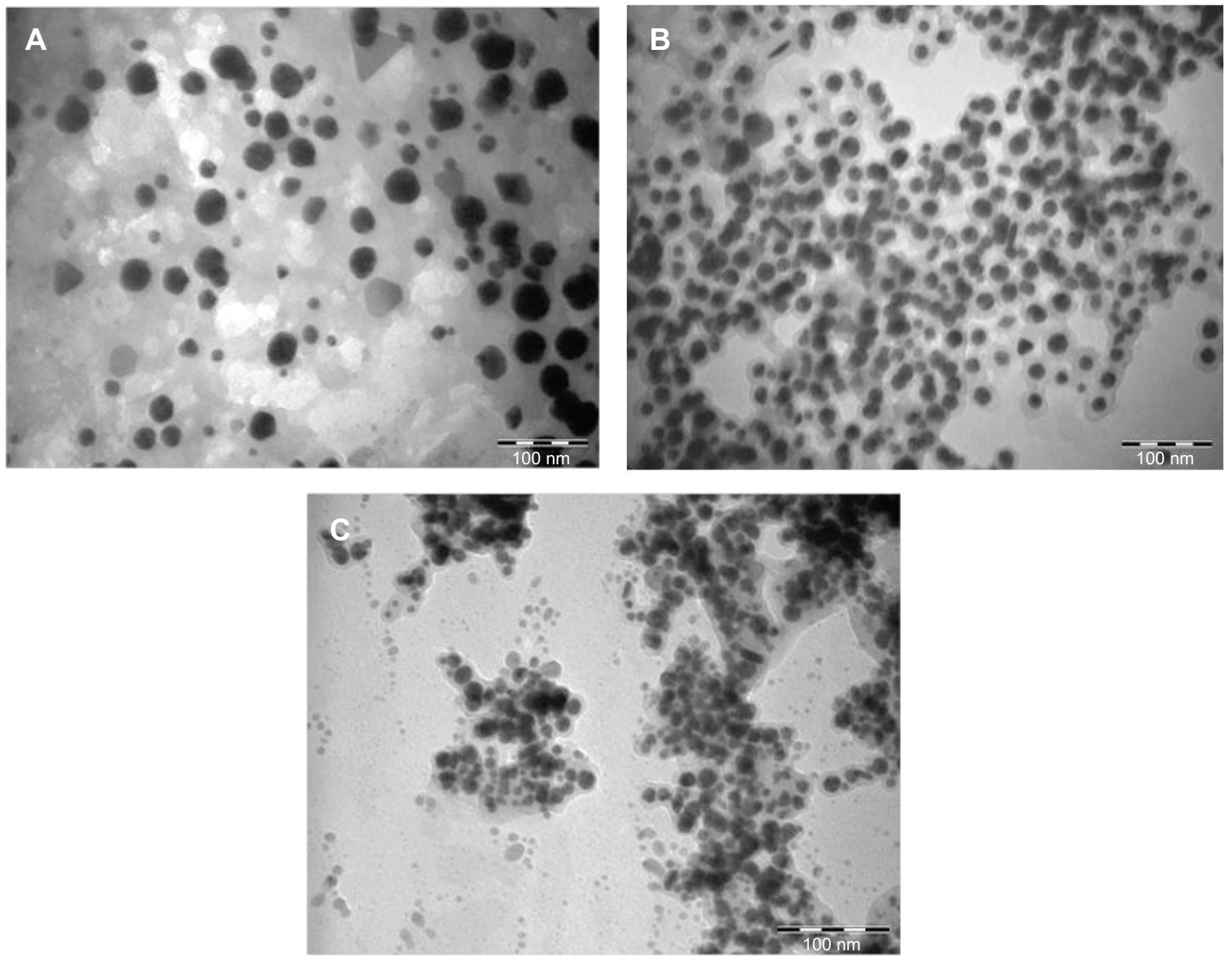

Figure 3 Transmission electron microscopy micrographs of (A) EGCG-, (B) RSV-, and (C) FS-capped GNPs. Note: Distances expressed in nanometers (nm).

Abbreviations: EGCG, epigallocatechin-3-gallate; RSV, resveratrol; FS, fisetin; GNPs, gold nanoparticles. 
Table I Mean diameter of EGCG-, RSV-, and FS-capped GNPs

\begin{tabular}{ll}
\hline $\begin{array}{l}\text { Polyphenol-capped } \\
\text { GNPs }\end{array}$ & $\begin{array}{l}\text { Mean diameter } \pm \text { standard } \\
\text { deviation }(\mathbf{n m})\end{array}$ \\
\hline EGCG-GNPs & $25.55 \pm 7.26^{*}$ \\
RSV-GNPs & $14.55 \pm 2.20$ \\
FS-GNPs & $9.76 \pm 1.56^{*}$ \\
\hline
\end{tabular}

Notes: Diameter expressed in nanometers $(\mathrm{nm})$. *Significantly different from other GNP-polyphenols. EGCG-GNPs, RSV-GNPs, and FS-GNPs indicate polyphenolcapped nanogold.

Abbreviations: EGCG, epigallocatechin-3-gallate; RSV, resveratrol; FS, fisetin; GNPs, gold nanoparticles.

observed (Figure 3B and C, respectively). As reported in Table 1, the size of the NPs was in the range of 10-25 nm and was influenced by the polyphenol used in the synthetic procedure.

These findings suggest that EGCG, RSV, and FS could be successfully used as reducing and stabilizing agents to produce GNPs with a narrow size distribution. Significant differences $(P<0.05)$ were found in mean diameter of EGCG-GNPs with respect to FS-GNPs.

The powder XRD patterns (Figure 4) showed that synthesized GNPs were crystalline. The pattern presents four distinct diffraction peaks at 38.17, 44.33, 64.70, and 77.70, which are assigned to (1 111$),\left(\begin{array}{lll}2 & 0 & 0\end{array}\right),\left(\begin{array}{lll}2 & 2 & 0\end{array}\right)$, and ( $\left.\begin{array}{lll}3 & 1 & 1\end{array}\right)$ crystalline plane diffraction peaks of cubic GNPs, respectively, in agreement with the database of the Joint Committee on Powder Diffraction Standards (JCPDS No 04-0784). The absence of any other crystallographic impurities peaks in the XRD spectrum confirmed the high purity of synthesized GNPs, in accordance with that reported earlier for similar prototypes. ${ }^{48}$

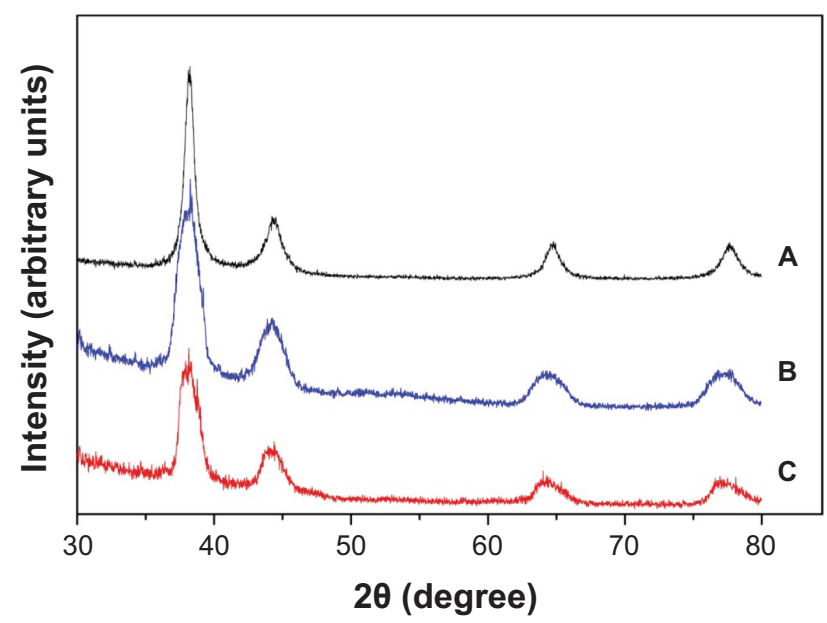

Figure 4 X-ray diffraction pattern of (A) EGCG-, (B) RSV-, and (C) FS-capped GNPs. Note: Diffraction angle $2 \theta$ expressed in degrees.

Abbreviations: EGCG, epigallocatechin-3-gallate; RSV, resveratrol; FS, fisetin; GNPs, gold nanoparticles.
The average particle size was estimated using the Scherrer equation:

$$
\mathrm{d}=(\mathrm{k} \lambda / \beta 1 / 2 \cos \theta)
$$

where $\mathrm{k}$ is the Scherrer constant (0.89), $\lambda$ is the $\mathrm{X}$-ray wavelength of $\mathrm{Cu} \mathrm{K} \alpha 1$ radiation, $\beta 1 / 2$ is the width of the XRD peak at half-height, and $\theta$ is the Bragg angle.

The average size of the NPs, calculated from the width of the diffraction peak, was about 19.49,12.16, and $9.90 \mathrm{~nm}$ for EGCG-GNPs, RSV-GNPs, and FS-GNPs, respectively, which were in line with the results obtained from TEM measurements.

To acquire further information about the presence of polyphenols coating in the NP surface, FT-IR measurements of both free polyphenols and the corresponding gold-capped derivatives were assessed.

The analysis of FT-IR spectrum of free compounds and corresponding capped GNPs evidenced an overlapping behavior between all three couples of samples (Figure 5). In particular, the spectra of EGCG and EGCG-GNPs are characterized by the typical $\mathrm{C}=\mathrm{O}$ ester band at about $1,700 \mathrm{~cm}^{-1}$ (Figure 5A). Moreover, aromatic double-bond stretching at about $1,610 \mathrm{~cm}^{-1}$, aromatic $\mathrm{C}-\mathrm{C}$ stretching at $1,450 \mathrm{~cm}^{-1}$, $\mathrm{O}-\mathrm{H}$ bending vibration centered at $1,375 \mathrm{~cm}^{-1}$, and $\mathrm{C}-\mathrm{O}$ stretching at $1,210 \mathrm{~cm}^{-1}$ were also detected in both samples. Spectra of RSV and RSV-GNPs (Figure 5B) revealed four intense bands at about 1,610,1,590,1,450, and $1,150 \mathrm{~cm}^{-1}$, corresponding to $\mathrm{C}-\mathrm{C}$ aromatic double-bond stretching, $\mathrm{C}-\mathrm{C}$ olefinic stretching, aromatic $\mathrm{C}-\mathrm{H}$ stretching, and $\mathrm{C}-\mathrm{C}$ aromatic bending vibration, respectively. Finally, FS and its nanogold derivative showed similar pattern signals (Figure 5C), which are constituted by $\mathrm{C}=\mathrm{O}$ stretching of the ketone functionality located on the chromen-4-one ring $(1,725-1,690$ $\left.\mathrm{cm}^{-1}\right), \mathrm{C}=\mathrm{C}$ stretching $\left(1,605 \mathrm{~cm}^{-1}\right), \mathrm{C}-\mathrm{C}$ stretching $(1,260$ $\left.\mathrm{cm}^{-1}\right)$, and $\mathrm{C}-\mathrm{OH}$ bending vibration $\left(1,110 \mathrm{~cm}^{-1}\right)$.

\section{Determination of polyphenol content}

Since GNPs can be proposed as suitable carriers for the delivery of bioactive EGCG, RSV, and FS, as well as for other related phytochemicals, it is important to determine the amount of polyphenols adsorbed on the NPs' surface.

To evaluate the EGCG, RSV, and FS content in gold nanosystems, the rapid colorimetric Folin-Ciocalteu method, frequently used for quantification of total phenolic content in natural products, was considered in this study. ${ }^{49} \mathrm{We}$ derived the concentration of each polyphenol in NPs from the standard curve prepared using EGCG, RSV, and FS raw materials 
A

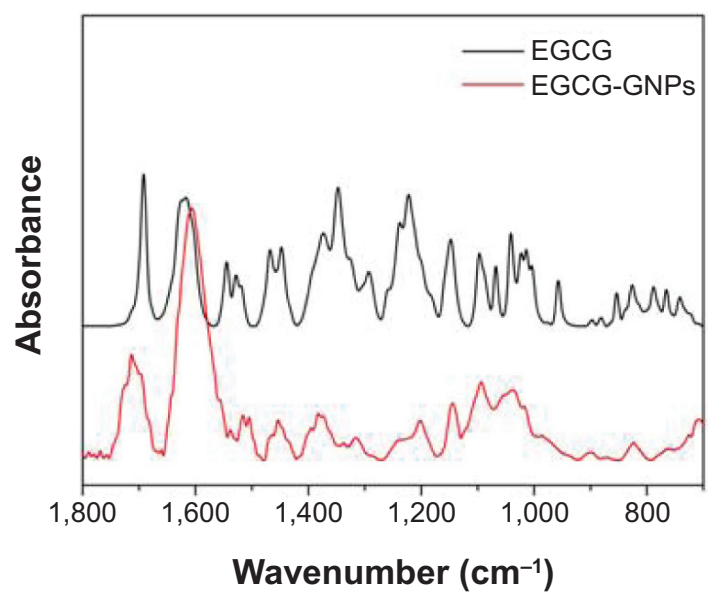

B

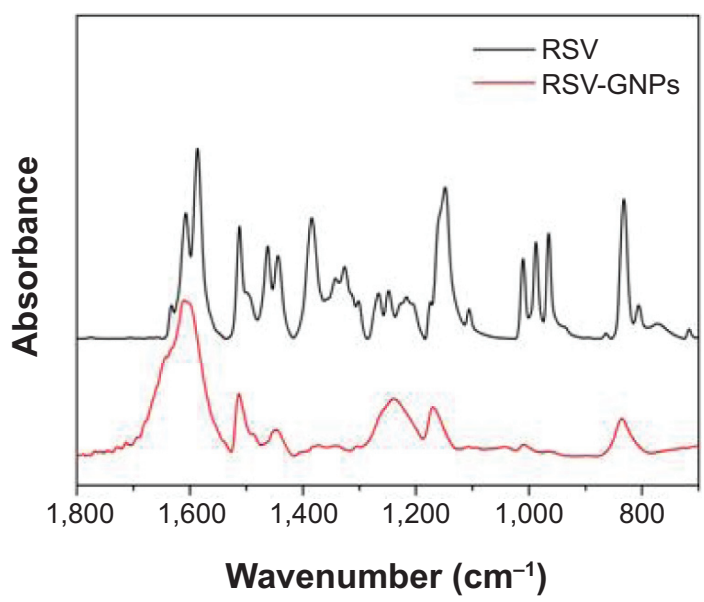

C

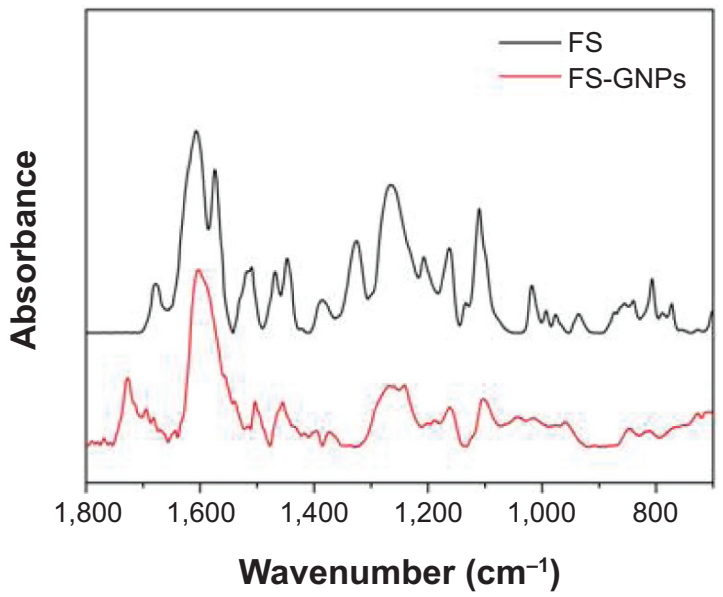

Figure 5 Fourier transform infrared spectra of (A) EGCG-GNPs, (B) RSV-GNPs, and (C) FS-GNPs, compared with pure EGCG, RSV, and FS, respectively. Note: Wavenumber expressed in $\mathrm{cm}^{-1}$.

Abbreviations: EGCG, epigallocatechin-3-gallate; RSV, resveratrol; FS, fisetin; GNPs, gold nanoparticles.

(Figure S2). The percentage of polyphenol content resulted as $2.71 \%, 3.23 \%$, and $5.47 \%(\mathrm{w} / \mathrm{w})$ for GNPs synthesized using EGCG, RSV, and FS, respectively.

\section{In vitro stability}

One of the most important aspects to be taken into account for various biomedical applications of GNPs is their stability in physiological media, in order to prevent aggregation. ${ }^{32}$ In fact, it represents a key issue that underlies multiple deleterious effects in many therapeutics, including loss of efficacy, altered pharmacokinetics, and induction of unwanted immunogenicity.

The stability of polyphenol-capped GNPs was monitored using absorption spectroscopy in $0.5 \%$ BSA, $0.5 \%$ cysteine, and $5.0 \% \mathrm{NaCl}$ solutions over 3 hours. We have also investigated the stability of GNPs in PBS at pH 5, 7, and 9.
On the whole, the plasmon wavelengths in all the tested GNPs (Figure 6A-C) show minimal shifts of $1-5 \mathrm{~nm}$, confirming that the NPs are intact, thus demonstrating good in vitro stability in simulated biological fluids. These results confirm that EGCG, RSV, and FS are acting in stabilizing gold nanoparticulate systems from agglomerations in solution. It was also observed that at $\mathrm{pH} 9$ all GNPs are characterized by a more remarkable $(7-12 \mathrm{~nm})$ blue shift in SPR band. As previously reported, the shift in SPR can be correlated with NPs' surface charge at different $\mathrm{pH} .{ }^{50} \mathrm{In}$ alkaline $\mathrm{pH}$, the presence of a high concentration of hydroxyl ions on the surface of NPs might maximize their repulsive electrostatic and electrosteric interactions, thus reducing particles aggregation, as detected by the hypsochromic shift of UV-vis peak. ${ }^{48}$

On the other hand, when colloidal gold solution was added into BSA, the position of the peak of colloidal gold 
A

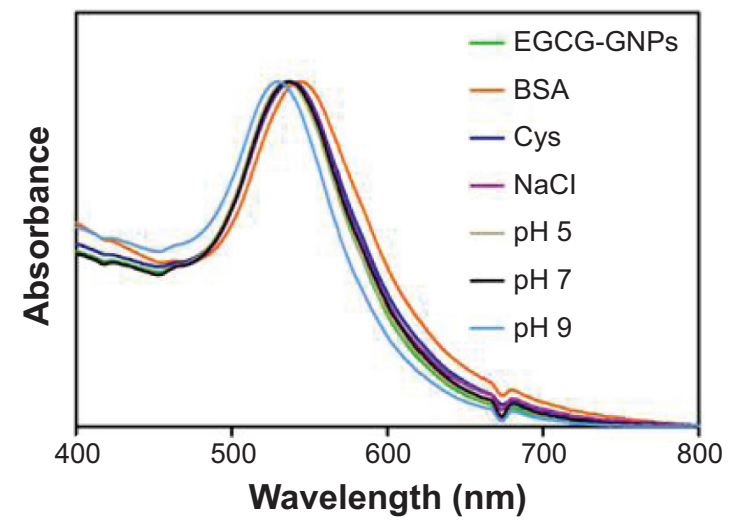

B

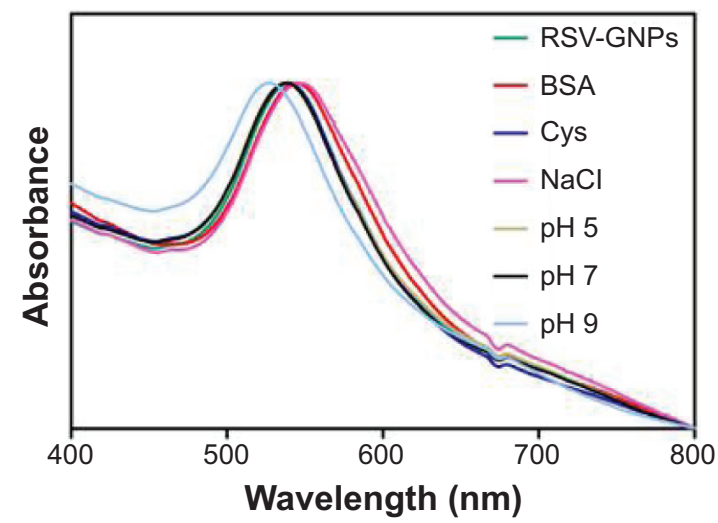

C

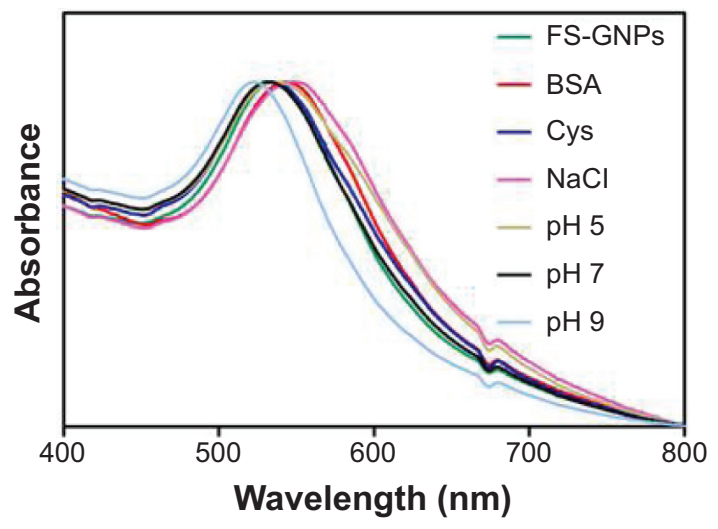

Figure 6 In vitro stability ultraviolet-visible studies of GNPs capped with (A) EGCG, (B) RSV, and (C) FS in different media. Notes: Wavelength expressed in nm. EGCG-GNPs, RSV-GNPs, and FS-GNPs indicate polyphenol-capped nanogold.

Abbreviations: EGCG, epigallocatechin-3-gallate; RSV, resveratrol; FS, fisetin; GNPs, gold nanoparticles; BSA, bovine serum albumin; Cys, cysteine; NaCl, sodium chloride.

slightly shifted red $(\sim 7 \mathrm{~nm})$. These results could be attributed to the possible formations of BSA-colloidal gold complex, probably due to the interaction with free thiol groups of protein. ${ }^{51}$

Concerning RSV-GNPs and FS-GNPs, a slight red shift (7 and $10 \mathrm{~nm}$, respectively) was also observed when the strong electrolyte (ie, $\mathrm{NaCl}$ ) was added at $5.0 \%$ concentration; the high concentration of ions presumably shielded the repulsive electrostatic forces between NPs, allowing them to form small aggregates. Considering that in physiological conditions the concentration of $\mathrm{NaCl}$ corresponds to $0.9 \%$ (150 mM), all produced GNPs can be considered stable.

\section{Antioxidant activity: DPPH assay}

Measurement of radical-scavenging activity using discoloration of DPPH has been widely used due to its stability, simplicity, and reproducibility. ${ }^{42}$

As shown in Figure 7A, all GNPs scavenged DPPH radicals in a dose-dependent manner and shared a good linear correlation between the percentage inhibition of the DPPH and the amount of GNPs used (ECGG-GNPs: $R^{2}=0.9534$; RSV-GNPs: $R^{2}=0.9295$; FS-GNPs: $\left.R^{2}=0.9880\right)$.

At all tested concentrations, EGCG-GNPs and FS-GNPs exhibited a similar DPPH-scavenging activity. Percentage inhibition of DPPH ranged from about $16 \%$ and $19 \%$ at a lower concentration $(25 \mu \mathrm{g} / \mathrm{mL})$ to $60 \%$ and $66 \%$ obtained at higher concentration $(200 \mu \mathrm{g} / \mathrm{mL})$ for EGCG-GNPs and FSGNPs, respectively. On the contrary, the RSV-GNPs showed a significantly lower $(P<0.05)$ free radical-scavenging effect when compared with EGCG-GNPs and FS-GNPs, with only $26 \%$ of scavenging activity at $200 \mu \mathrm{g} / \mathrm{mL}$.

Moreover, the kinetics of DPPH• radicals-scavenging activity of all GNPs increased with an increase in the concentration, and the decrease in absorbance reached a plateau within the 5-minute sampling period, which indicated that the reaction had reached completion (data not shown).

\section{Antioxidant activity: ABTS assay}

The ABTS assay is another commonly used method available to evaluate antioxidant activities of natural compounds 
A

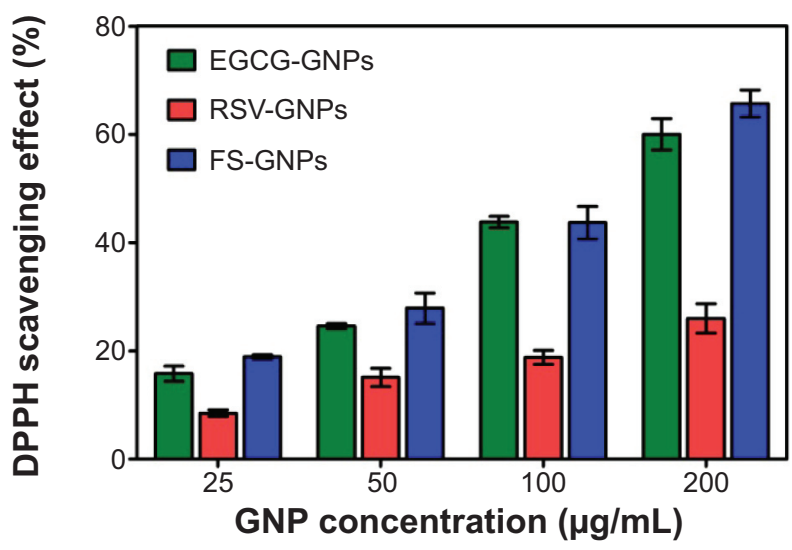

B

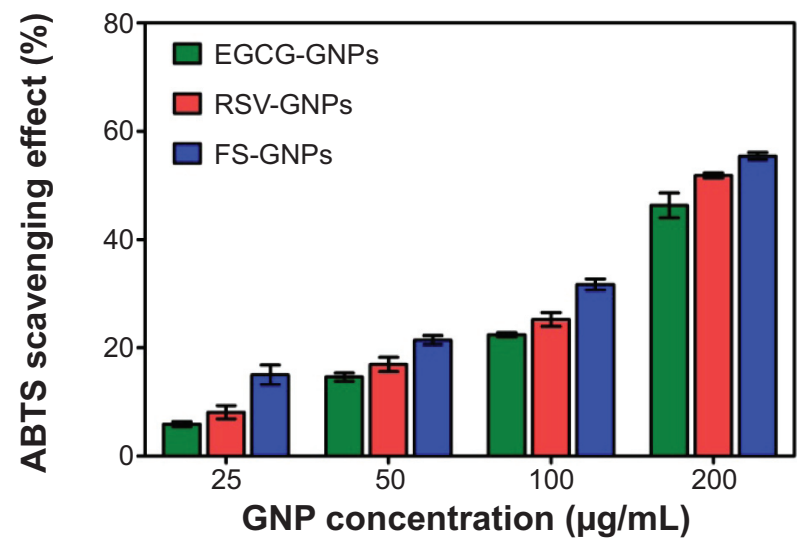

Figure 7 Antioxidant activities of EGCG-GNPs, RSV-GNPs, and FS-GNPs at various concentrations assessed by percentage inhibition of DPPH (A) and ABTS (B) radicals. Notes: GNP concentration expressed in $\mu \mathrm{g} / \mathrm{mL}$. EGCG-GNPs, RSV-GNPs, and FS-GNPs indicate polyphenol-capped nanogold.

Abbreviations: EGCG, epigallocatechin-3-gallate; RSV, resveratrol; FS, fisetin; GNPs, gold nanoparticles; DPPH, 2,2-diphenyl-I-picrylhydrazyl; ABTS, 2,2'-azinobis (3-ethylbenzothiazoline-6-sulfonic acid).

based on their ability to scavenge the long-life radical cation $\mathrm{ABTS} \bullet+.{ }^{52}$

The results reported in Figure 7B show that radicalscavenging activities of all the GNPs increased linearly with increasing concentration, exhibiting their dose-dependent nature (EGCG-GNPs: $R^{2}=0.9921$; RSV-GNPs: $R^{2}=0.9924$; FS-GNPs: $\left.R^{2}=0.9993\right)$. The antioxidant activity was found to be stronger for FS-GNPs, followed by the RSV-GNPs and then EGCG-GNPs.

The scavenging percentages range from $6 \%, 8 \%$, and $15 \%$ at $25 \mu \mathrm{g} / \mathrm{mL}$ to $46 \%, 51 \%$, and $55 \%$ at higher concentration $(200 \mu \mathrm{g} / \mathrm{mL})$ for EGCG-GNPs, RSV-GNPs, and FS-GNPs, respectively. Interestingly, at all tested concentrations, the reaction reaches steady state conditions after the first 2 minutes (data not shown), suggesting that polyphenols on the surface of GNPs maintained a high antioxidant efficiency.

To elucidate in more detail the antioxidant activity differences detected for the obtained GNPs, the DPPH- and ABTSscavenging effects of EGCG, RSV, and FS raw materials at different concentrations were also evaluated.

As shown in Figure S3, the percentage of inhibition for DPPH of all polyphenols showed a good linearity at the tested concentrations. EGCG exhibited the highest antioxidant capacity with a percentage of DPPH inhibition that ranged from $9.5 \%(0.25 \mu \mathrm{g} / \mathrm{mL})$ to $66 \%(2.5 \mu \mathrm{g} / \mathrm{mL})$. At the same concentrations, values found for FS were decreased and ranged from $8.5 \%$ to $60 \%$. On the other hand, RSV showed the lowest antioxidant potential with comparable activity at higher concentrations ( $62.1 \%$ inhibition of DPPH at $20 \mu \mathrm{g} / \mathrm{mL})$.

These findings are in agreement with previous reports and suggest that the DPPH antioxidant activity of polyphenolic compounds is closely associated with their chemical structure, such as the number and position of phenolic hydrogen, which influences the accessibility to the radical centre of $\mathrm{DPPH} \bullet$. Thus, the greater number of hydroxyl groups, especially $O$-dihydroxy group on B-ring of EGCG and FS with respect to RSV, are potentially able to quench more efficiently the free radicals by forming resonance-stabilized phenoxyl radicals. ${ }^{52}$

As concerns ABTS-scavenging activity (Figure S4), no significant differences were found for EGCG and RSV, with the percentage of inhibition of about $20 \%$ at lower concentration $(0.5 \mu \mathrm{g} / \mathrm{mL})$ and $65 \%$ at higher concentration $(10 \mu \mathrm{g} / \mathrm{mL})$. In contrast, the ABTS antioxidant activity of FS, at the same concentrations, decreased significantly, ranging from $16 \%$ to $53 \%$.

The different behavior of polyphenols, and in particular of RSV, observed in DPPH and ABTS assays suggests that the reaction mechanisms involved in both methods are influenced by the chemical structure of the antioxidants, and that the two assay cations react by using different active chemical groups. In particular, in contrast to the oxidation of polyphenols by DPPH radicals that occur at B-ring, forming the corresponding $\mathrm{O}$-quinone, or even at the $\mathrm{C}-2$ position of the C-ring, the oxidation of polyphenols by ABTS radical cations occurs at the A-ring. ${ }^{53}$

Additionally, no significant DPPH and ABTS antioxidant activities were detected by using as control the nonfunctionalized GNPs at the same concentration of polyphenols-capped GNPs, thus confirming that radical-scavenging activities are due to the presence of polyphenols on the GNPs' surface (data not shown). 


\section{Correlations between polyphenol content and antioxidant activity}

The correlation coefficients $\left(R^{2}\right)$ were used to evaluate the correlation between each polyphenol content in GNPs and the methods used to determine their antioxidant capacity. As reported in Figure S5, the $R^{2}$ values between DPPH and polyphenolic content were $0.9654,0.9578$, and 0.9944 for EGCG-GNPs, RSV-GNPs, and FS-GNPs, respectively. High correlation was also demonstrated between ABTS and polyphenolic content $\left(R^{2}=0.9944,0.9923\right.$, and 0.9961 for EGCGGNPs, RSV-GNPs, and FS-GNPs, respectively).

These results suggest that the higher DPPH and ABTS antioxidant activities are correlated to the high EGCG, RSV, and FS content in GNPs. The $R^{2}$ values for correlations between DPPH and ABTS assays were $R^{2}=0.9335,0.9374$, and 0.9843 for EGCG-GNPs, RSV-GNPs, and FS-GNPs, respectively (Figure S6).

\section{Antiproliferative activity and apoptosis activation in SH-SY5Y-CFP-DEVD-YFP cells}

Several polyphenols have shown remarkable cancer-preventive and some cancer-therapeutic effects, partially due to their ability to induce apoptosis in cancer cells without affecting normal cells.

In this scenario, growth-suppressive effect on cell proliferation demonstrated by polyphenols such as EGCG is mediated through programmed cell death or apoptosis by interfering with many cellular processes, including nuclear condensation, caspase- 3 activation, and poly-ADP ribose polymerase cleavage, as well as eliciting the production of intracellular reactive oxygen species. ${ }^{54}$ The essential role of caspases (in particular, the key caspase-3 protein) in EGCGmediated inhibition of NF-kB and its subsequent apoptosis has also been demonstrated..$^{55}$

To assess whether the engineered GNPs may alter cell proliferation and induce apoptosis, we took advantage of the neuronal SH-SY5Y cells stably expressing CFP-DEVD-YFP reporter. ${ }^{56}$ In this reporter the sequence coding for CFP (CFP: GFP with mutations Y66W, F64L, S65T, N146I, M153T, and V163A) is fused to the one coding for YPF (YFP: a particular GFP variant called Venus with mutations F64L, M153T, V163A, and S175G) via the aminoacidic sequence DEVD, which is the substrate for active caspase-3. Different stable cell lines were generated and characterized for the first time. Figure 8 shows the SH-SY5Y stable cell line used in the following experiments. The expression levels of CFP-DEVDYFP were evaluated by Western blot (Figure 8A) and immunofluorescence (Figure 8B) experiments after induction of apoptosis by staurosporine treatment. As shown in Figure 8A, treatment with staurosporine determined the cleavage of DEVD target sequence and the resulting separation of two fluorescent proteins, indicating the activation of caspase-3.

Next, SH-SY5Y-CFP-DEVD-YFP cells were treated for 72 hours with EGCG-GNPs at different concentrations and cell viability was measured by MTS assay (Figure 8C). At the end of 72 hours of exposure, EGCG-GNPs decreased cell viability approximately in a dose-dependent manner, displaying an overlapping behavior with that of free EGCG. More specifically, both EGGC-GNPs and free EGCG exhibited similar cytotoxicity profiles, showing a dose-dependent antiproliferative effect and inhibition values in the overall cell growth. With respect to control, at $1 \mu \mathrm{g} / \mathrm{mL}$ concentration, EGCG-GNPs determined a growth inhibition of SH-SY5Y-CFP-DEVD-YFP cells of $\sim 10 \%$, similarly to an equivalent amount of free EGCG (ie, cells resulted in being exposed to equimolar amounts of free and capped EGCG). At $5 \mu \mathrm{g} / \mathrm{mL}, 10 \mu \mathrm{g} / \mathrm{mL}$, and $25 \mu \mathrm{g} / \mathrm{mL}$ concentration exposure, tested EGCG-GNPs showed a reduction of cell viability of about $20 \%-30 \%$, and, finally, treatment of SHSY5Y-CFP-DEVD-YFP cells with $50 \mu \mathrm{g} / \mathrm{mL}$ of EGCGGNPs proved the highest cytotoxicity, showing a cell growth inhibition of $\sim 40 \%$ (Figure $8 \mathrm{C}$ ).

Moreover, treatment of cells with EGCG-GNPs (and free ECGG) shared increased CFP-DEVD-YFP reporter cleavage (Figure 8D) due to caspase-3 activation, thus supporting an apoptotic mechanism of action.

In summary, EGCG-GNPs induce a dose-dependent reduction on cell viability that is likely to involve the activation of the apoptotic pathways.

Of course, to evaluate whether these activities may have an in vivo relevance when providing a reasonable dose of these NPs, further studies are in progress to explore a large panel of more responsive tumor cells. However, for comparative purposes, in a previous in vivo study on polymeric NP-mediated delivery of bioactive catechins administered at concentrations ranging 5-20 $\mu \mathrm{g} /$ mouse, the polyphenols loading of NPs resulted in $3.38 \%$ and $5.76 \%$ of the polymer weight, similar to the concentration of polyphenols found in our prototypes. ${ }^{57}$

\section{Conclusion}

In this work, green synthesis of GNPs using natural polyphenols EGCG, RSV, and FS was successfully investigated and proposed as a simple, reproducible, low-cost, and nontoxic method. Stable nanogolds were uniformly generated and fully characterized for their physicochemical properties. Overall, enhanced antioxidant properties were detected for all three 
A

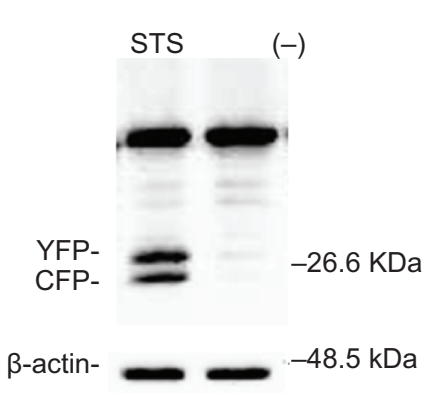

B

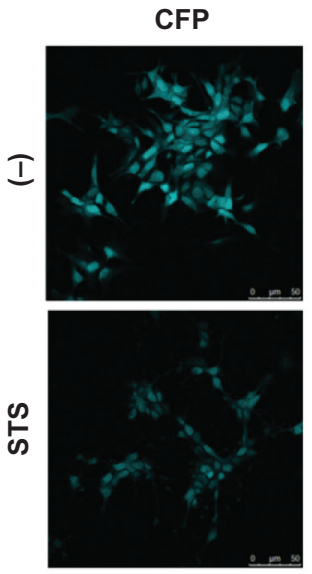

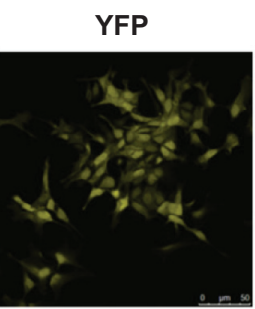

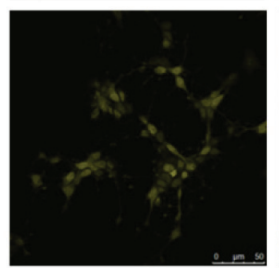

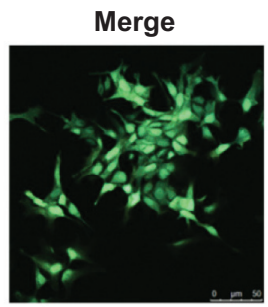
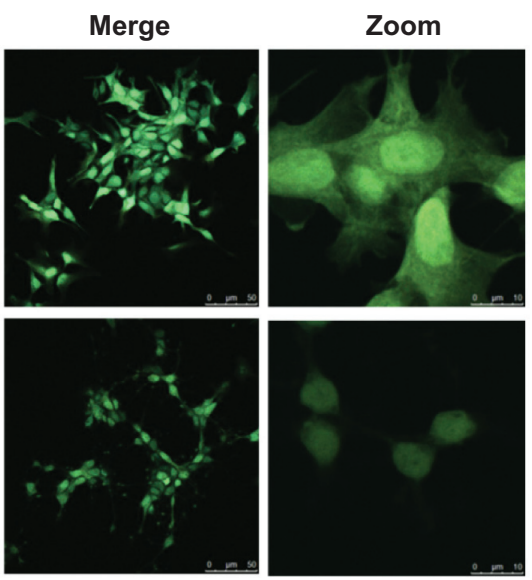

C

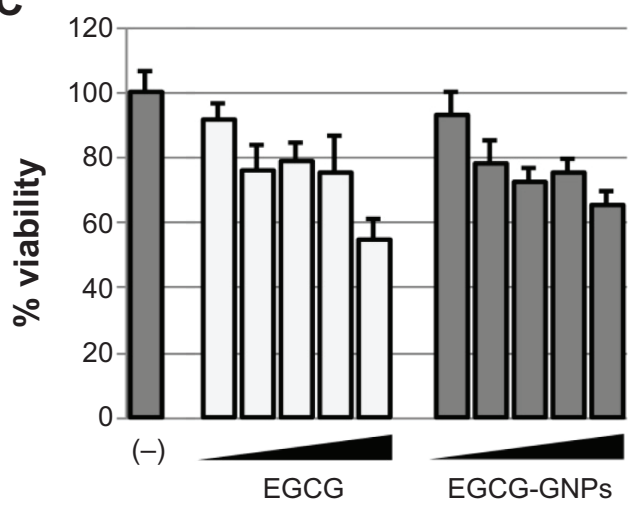

D

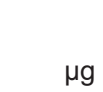

EGCG EGCG-GNPS

YFP-
CFPSTS $10 \quad 50$ 10 50 $(-)$

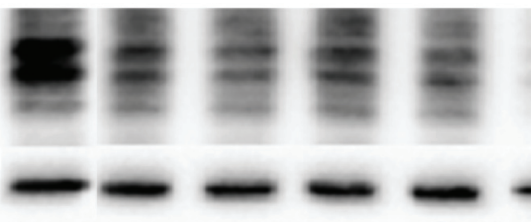

$\beta$-actin-

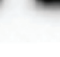

$-26.6 \mathrm{KDa}$

$-48.5 \mathrm{KDa}$

Figure 8 Effect of EGCG-GNPs on CFP-DEVD-YFP reporter gene. (A) SH-SY5Y-derived cells expressing CFP-DEVD-YFP reporter gene were left untreated (-) or treated $(+)$ for 2 hours with I $\mu \mathrm{M}$ staurosporine to induce caspase-3 activation. Cell lysates were subjected to reducing sodium dodecyl sulfate-polyacrylamide gel electrophoresis. The anti-GFP antibody was used to visualize CFP-DEVD-YFP reporter and anti- $\beta$-actin serves as control for equal loading of samples. (B) Autofluorescence analysis of cells as in (A). (C) Effect EGCG-GNPs at I, 5, 10, 25, and $50 \mu \mathrm{g} / \mathrm{mL}$ on SH-SY5Y-CFP-DEVD-YFP cells. (D) Western blots on protein extracts from cells treated as in (C) were performed using anti-GFP antibody and anti- $\beta$-actin as controls for equal EGCG loading of samples (EGCG-GNPs concentration $=10$ and $50 \mu g / m L$ ).

Notes: Results for MTS assays are the means \pm SEM of three experiments performed in triplicate. Concentration of free EGCG and EGCG capped in GNPs is the same. Abbreviations: EGCG, epigallocatechin-3-gallate; RSV, resveratrol; FS, fisetin; GNPs, gold nanoparticles; GFP, green fluorescent protein; MTS, 3(4,5-dimethylthiazol-2yl)-5(3-carboxymethoxyphenyl)-2-(4-sulfophenyl)-2H-tetrazolium; SEM, standard error of the mean.

prototypes, and this correlates well with the high EGCG, RSV, and FS content in GNPs. However, some differences in free radical-scavenging action between the three nanosystems have been observed, due to their peculiar chemical structure. Moreover, a high degree of correlation among the antioxidant activities measured by DPPH and ABTS assays has been found in these polyphenol-capped GNPs. Our data on the antiproliferative activity of EGCG-GNPs in a neuroblastoma cell model demonstrated that these constructs induce a concentration-dependent reduction on cell viability by involving the activation of the apoptotic pathways. Moreover, the effectiveness of EGCG in terms of antiproliferative efficacy can be retained in EGCG-capped GNPs and supports the antioxidant capacity of these polyphenols and their ability to catalyze the decomposition/scavenging of free radicals.

These results would make these stable polyphenol-capped gold nanoprototypes suitable as functional and promising candidates for the treatment of disorders associated with oxidative stress, and extensive preclinical studies are warranted.

\section{Acknowledgments}

The authors gratefully acknowledge the Regione Autonoma della Sardegna for financial support of Grant No CRP-25920, "Development of novel targeted nanodevices for the prevention, diagnosis and treatment of prostate cancer", awarded to MS within the frame of "Legge regionale n. 7/2007, promozione della ricerca scientifica e dell'innovazione tecnologica in Sardegna, Annualità 2010". This work was also supported by Fondazione Banco di Sardegna (Grant Nos 2013-1479 and 2013.0325) and Regione Sardegna (Grant No CRP-17171).

\section{Disclosure}

The authors report no conflicts of interest in this work. 


\section{References}

1. Sperling RA, Rivera-Gil P, Zhang F, Zanella M, Parak WJ. Biological applications of gold nanoparticles. Chem Soc Rev. 2008;37(9): 1896-1908.

2. Dykman L, Khlebtsov N. Gold nanoparticles in biomedical applications: recent advances and perspectives. Chem Soc Rev. 2012;41(6): 2256-2282.

3. Dreaden EC, Alkilany AM, Huang X, Murphy CJ, El-Sayed MA. The golden age: gold nanoparticles for biomedicine. Chem Soc Rev. 2012; 41(7):2740-2779.

4. Vigderman L, Zubarev ER. Therapeutic platforms based on gold nanoparticles and their covalent conjugates with drug molecules. Adv Drug Deliv Rev. 2013;65(5):663-676.

5. Thomas M, Klibanov AM. Conjugation to gold nanoparticles enhances polyethylenimine's transfer of plasmid DNA into mammalian cells. Proc Natl Acad Sci US A. 2003;100(16):9138-9143.

6. Du J, Jin J, Yan M, Lu Y. Synthetic nanocarriers for intracellular protein delivery. Curr Drug Metab. 2012;13(1):82-92.

7. Giljohann DA, Seferos DS, Daniel WL, Massich MD, Patel PC, Mirkin CA. Gold nanoparticles for biology and medicine. Angew Chem Int Ed Engl. 2010;49(19):3280-3294.

8. Llevot A, Astruc D. Applications of vectorized gold nanoparticles to the diagnosis and therapy of cancer. Chem Soc Rev. 2012;41(1): 242-257.

9. Cai W, Gao T, Hong H, Sun J. Applications of gold nanoparticles in cancer nanotechnology. Nanotechnol Sci Appl. 2008;1:17-32.

10. Turkevich J, Stevenson PC, Hillier J. A study of the nucleation and growth processes in the synthesis of colloidal gold. Discuss Faraday Soc. 1951;11:55-75.

11. Daniel MC, Astruc D. Gold nanoparticles: assembly, supramolecular chemistry, quantum-size-related properties, and applications toward biology, catalysis, and nanotechnology. Chem Rev. 2004;104: 293-346.

12. Corbierre MK, Cameron NS, Lennox RB. Polymer-stabilized gold nanoparticles with high grafting densities. Langmuir. 2004; 20(7):2867-2873.

13. DeLong RK, Reynolds CM, Malcolm Y, Schaeffer A, Severs T, Wanekaya A. Functionalized gold nanoparticles for the binding, stabilization, and delivery of therapeutic DNA, RNA, and other biological macromolecules. Nanotechnol Sci App. 2010;3(1):53-63.

14. Chompoosor A, Han G, Rotello VM. Charge dependence of ligand release and monolayer stability of gold nanoparticles by biogenic thiols. Bioconjug Chem. 2008;19(7):1342-1345.

15. Katti K, Chanda N, Shukla R, et al. Green nanotechnology from cumin phytochemicals: generation of biocompatible gold nanoparticles. Int J Green Nanotechnol Biomed. 2009;1(1):B39-B52.

16. Menon D, Basanth A, Retnakumari A, Manzoor K, Nair SV. Green synthesis of biocompatible gold nanocrystals with tunable surface plasmon resonance using garlic phytochemicals. J Biomed Nanotechnol. 2012;8(6):901-911.

17. Mukherjee S, Sushma V, Patra S, et al. Green chemistry approach for the synthesis and stabilization of biocompatible gold nanoparticles and their potential applications in cancer therapy. Nanotechnology. 2012;23(45):455103.

18. Das S, Roy P, Mondal S, Bera T, Mukherjee A. One pot synthesis of gold nanoparticles and application in chemotherapy of wild and resistant type visceral leishmaniasis. Colloids Surf B Biointerfaces. 2013;107:27-34

19. Arunachalam KD, Annamalai SK, Hari S. One-step green synthesis and characterization of leaf extract-mediated biocompatible silver and gold nanoparticles from Memecylon umbellatum. Int J Nanomedicine. 2013;8:1307-1315.

20. Arunachalam KD, Annamalai SK. Chrysopogon zizanioides aqueous extract mediated synthesis, characterization of crystalline silver and gold nanoparticles for biomedical applications. Int J Nanomedicine. 2013;8:2375-2384.

21. Han X, Shen T, Lou H. Dietary polyphenols and their biological significance. Int J Mol Sci. 2007;8(9):950-988.
22. Scalbert A, Johnson IT, Saltmarsh M. Polyphenols: antioxidants and beyond. Am J Clin Nutr. 2005;81(1 Suppl):215S-217S.

23. Higdon JV, Frei B. Tea catechins and polyphenols: health effects, metabolism, and antioxidant functions. Crit Rev Food Sci Nutr. 2003; 43(1):89-143.

24. Khan N, Mukhtar H. Tea polyphenols for health promotion. Life Sci. 2007;81(7):519-533.

25. Pervaiz S, Holme AL. Resveratrol: its biologic targets and functional activity. Antioxid Redox Signal. 2009;11(11):2851-2897.

26. Khan N, Asim M, Afaq F, Abu Zaid M, Mukhtar H. A novel dietary flavonoid fisetin inhibits androgen receptor signaling and tumor growth in athymic nude mice. Cancer Res. 2008;68(20):8555-8563.

27. Siddiqui IA, Adhami VM, Bharali DJ, et al. Introducing nanochemoprevention as a novel approach for cancer control: proof of principle with green tea polyphenol epigallocatechin-3-gallate. Cancer Res. 2009;69(5):1712-1716.

28. Sanna V, Pintus G, Roggio AM, et al. Targeted biocompatible nanoparticles for the delivery of (-)-epigallocatechin 3-gallate to prostate cancer cells. J Med Chem. 2011;54(5):1321-1332.

29. Sanna V, Sechi M. Nanoparticle therapeutics for prostate cancer treatment. Nanomedicine. 2012;8:S31-S36.

30. Sanna V, Roggio AM, Siliani S, et al. Development of novel cationic chitosan- and anionic alginate-coated poly(D,L-lactide-co-glycolide) nanoparticles for controlled release and light protection of resveratrol. Int J Nanomedicine. 2012;7:5501-5516.

31. Sanna V, Siddiqui IA, Sechi M, Mukhtar H. Nanoformulation of natural products for prevention and therapy of prostate cancer. Cancer Lett. 2013;334:142-151.

32. Nune SK, Chanda N, Shukla R, et al. Green nanotechnology from tea: phytochemicals in tea as building blocks for production of biocompatible gold nanoparticles. J Mater Chem. 2009;19(19): 2912-2920.

33. Shukla R, Chanda N, Zambre A, et al. Laminin receptor specific therapeutic gold nanoparticles (198AuNP-EGCg) show efficacy in treating prostate cancer. Proc Natl Acad Sci U S A. 2012;109(31): 12426-12431.

34. Hsieh DS, Lu HC, Chen CC, Wu CJ, Yeh MK. The preparation and characterization of gold-conjugated polyphenol nanoparticles as a novel delivery system. Int J Nanomedicine. 2012;7:1623-1633.

35. Huang X, Wu H, Liao X, Shi B. One-step, size-controlled synthesis of gold nanoparticles at room temperature using plant tannin. Green Chem. 2010;12:395-399.

36. Wu H, Huang X, Gao M, Liao X, Shi B. Polyphenol-grafted collagen fiber as reductant and stabilizer for one-step synthesis of size-controlled gold nanoparticles and their catalytic application to 4-nitrophenol reduction. Green Chem. 2011;13:651-658.

37. Zhu H, Du ML, Zou ML, Xu CS, Li N, Fu YQ. Facile and green synthesis of well-dispersed $\mathrm{Au}$ nanoparticles in PAN nanofibers by tea polyphenols. J Mater Chem. 2012;22:9301-9307.

38. Singh DK, Jagannathan R, Khandelwal P, Abraham PM, Poddar P. In situ synthesis and surface functionalization of gold nanoparticles with curcumin and their antioxidant properties: an experimental and density functional theory investigation. Nanoscale. 2013;5:1882-1893.

39. Lowry OH, Rosebrough NJ, Farr AL, Randall RJ. Protein measurement with the folin phenol reagent. J Biol Chem. 1951;193:265-275.

40. George S, Brat P, Alter P, Amiot MJ. Rapid determination of polyphenols and vitamin C in plant-derived products. J Agric Food Chem. 2005;53(5):1370-1373.

41. Blois MS. Antioxidant determinations by the use of a stable free radical. Nature. 1958;181:1199-1200.

42. Gülçin I. Antioxidant properties of resveratrol: a structure-activity insight. Innov Food Sci Emerg Technol. 2010;11(1):210-218.

43. Re R, Pellegrini N, Proteggente A, Pannala A, Yang M, Rice-Evans C. Antioxidant activity applying an improved ABTS radical cation decolorization assay. Free Radic Biol Med. 1999;26(9-10):1231-1237.

44. Gülçin I, Elias R, Gepdiremen A, Boyer L. Antioxidant activity of lignans from fringe tree (chionanthus virginicus L.). Eur Food Res Technol. 2006;223(6):759-767. 
45. Baschong W, Suetterlin R, Laeng RH. Control of autofluorescence of archival formaldehyde-fixed, paraffin-embedded tissue in confocal laser scanning microscopy (CLSM). J Histochem Cytochem. 2001;49:1565-1572.

46. Iaccarino $\mathrm{C}$, Mura ME, Esposito S, et al. Bcl2-A1 interacts with procaspase-3: implications for amyotrophic lateral sclerosis. Neurobiol Dis. 2011;43:642-650.

47. Philip D. Synthesis and spectroscopic characterization of gold nanoparticles. Spectrochim Acta A Mol Biomol Spectrosc. 2008;71(1):80-85.

48. Philip D. Green synthesis of gold and silver nanoparticles using hibiscus rosa sinensis. Phys E. 2010;42(5):1417-1424.

49. Singleton VL, Orthofer R, Lamuela-Raventós RM. Analysis of total phenols and other oxidation substrates and antioxidants by means of folin-ciocalteu reagent. Methods Enzymol. 1998;299:152-178.

50. Dauthal $P$, Mukhopadhyay $M$. In-vitro free radical scavenging activity of biosynthesized gold and silver nanoparticles using prunus armeniaca (apricot) fruit extract nanomaterials in energy, health and environment. J Nanopart Res. 2013;1:15.

51. Gao D, Tian Y, Bi S, Chen Y, Yu A, Zhang H. Studies on the interaction of colloidal gold and serum albumins by spectral methods. Spectrochim Acta A Mol Biomol Spectrosc. 2005;62(4-5):1203-1208.

52. Rice-Evans CA, Miller NJ, Paganga G. Structure-antioxidant activity relationships of flavonoids and phenolic acids. Free Radic Biol Med. 1996;20(7):933-956.

53. Osman AM, Wong KKY, Fernyhough A. ABTS radical-driven oxidation of polyphenols: isolation and structural elucidation of covalent adducts. Biochem Biophys Res Comm. 2006;346:321-329.
54. Qanungo S, Das M, Haldar S, Basu A. Epigallocatechin-3-gallate induces mitochondrial membrane depolarization and caspase-dependent apoptosis in pancreatic cancer cells. Carcinogenesis. 2005;26: 958-967.

55. Islam $\mathrm{S}$, Islam N, Kermode $\mathrm{T}$, et al. Involvement of caspase-3 in epigallocatechin-3-gallate-mediated apoptosis of human chondrosarcoma cells. Biochem Biophys Res Commun. 2000;270;793-797.

56. Tyas L, Brophy VA, Pope A, Rivett AJ, Tavaré JM. Rapid caspase-3 activation during apoptosis revealed using fluorescence-resonance energy transfer. EMBO Rep. 2000;3:267-270.

57. Srivastava AK, Bhatnagar P, Singh M, et al. Synthesis of PLGA nanoparticles of tea polyphenols and their strong in vivo protective effect against chemically induced DNA damage. Int J Nanomedicine. 2013;8:1451-1462. 


\section{Supplementary materials}

Figures S1-S6 report details on photographs of colloidal EGCG-GNPs, calibration curves, and antioxidant activity of pure EGCG, RSV, and FS at various concentrations assessed by percentage inhibition of DPPH and ABTS, as well as correlation between radical-scavenging capacity assays (DPPH and ABTS) and correlation between DPPH and ABTS assays of nanogolds.

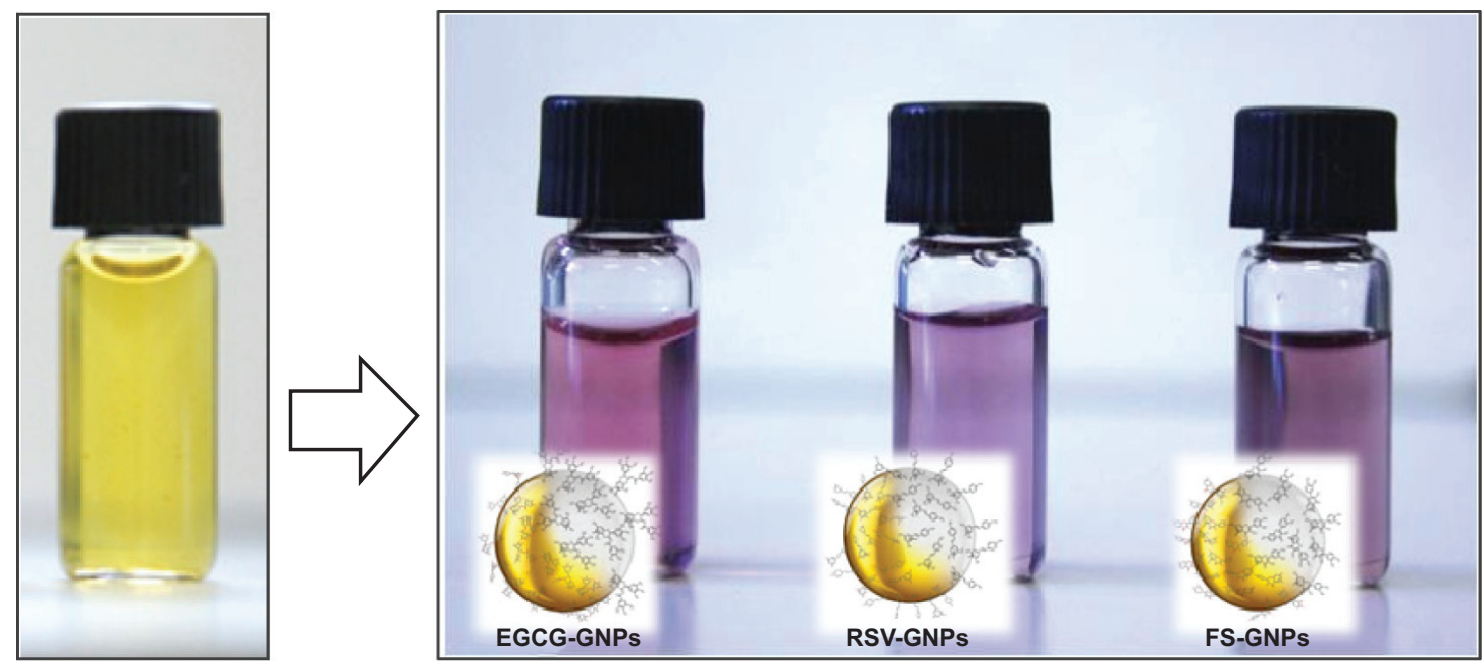

Figure SI Photograph of $\mathrm{HAuCl}_{4}$ solution (yellow solution) and colloidal EGCG-GNPs (left), RSV-GNPs (middle), and FS-GNPs (right). Abbreviations: EGCG, epigallocatechin-3-gallate; RSV, resveratrol; FS, fisetin; GNPs, gold nanoparticles.

A

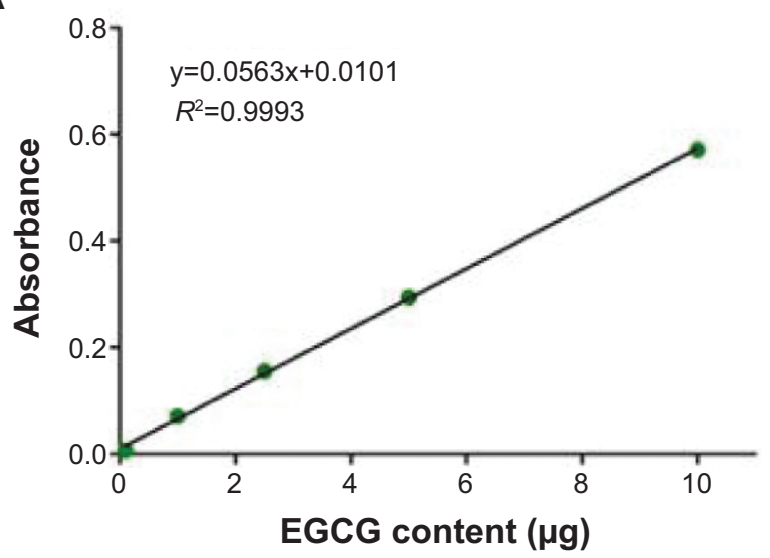

B

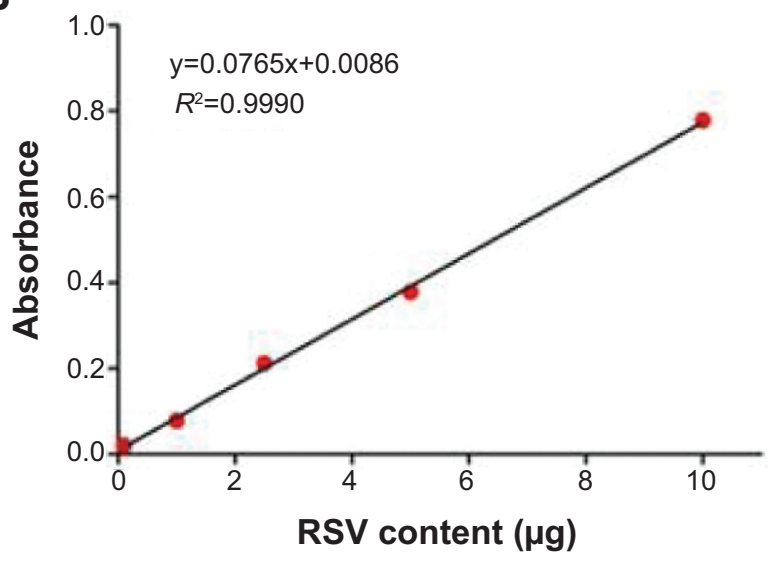

C

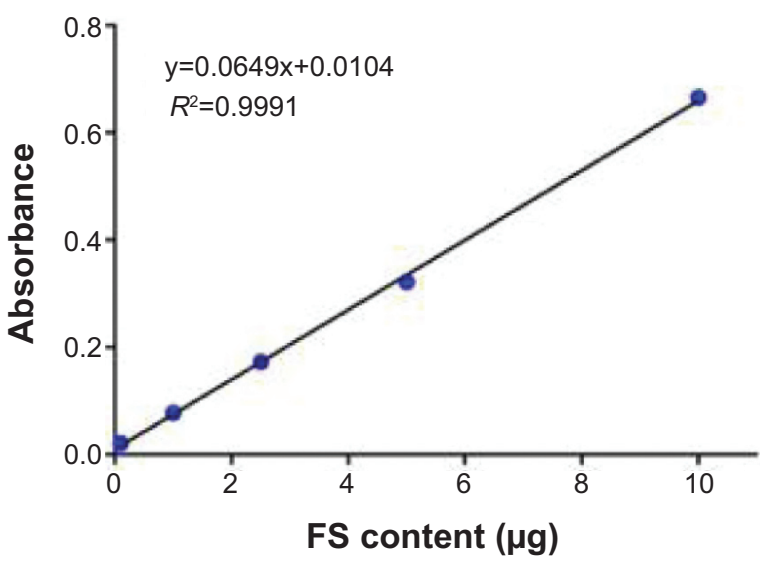

Figure S2 Calibration curves of pure (A) EGCG, (B) RSV, and (C) FS used for the determination of polyphenol content. Abbreviations: EGCG, epigallocatechin-3-gallate; RSV, resveratrol; FS, fisetin. 
A

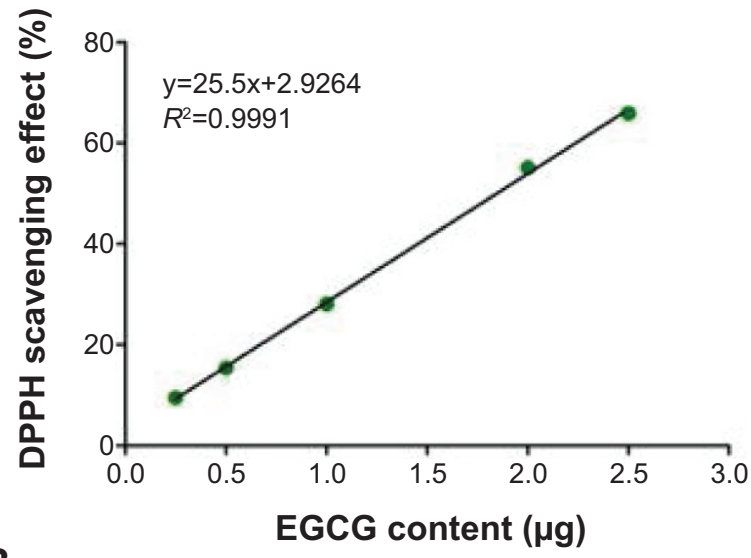

B

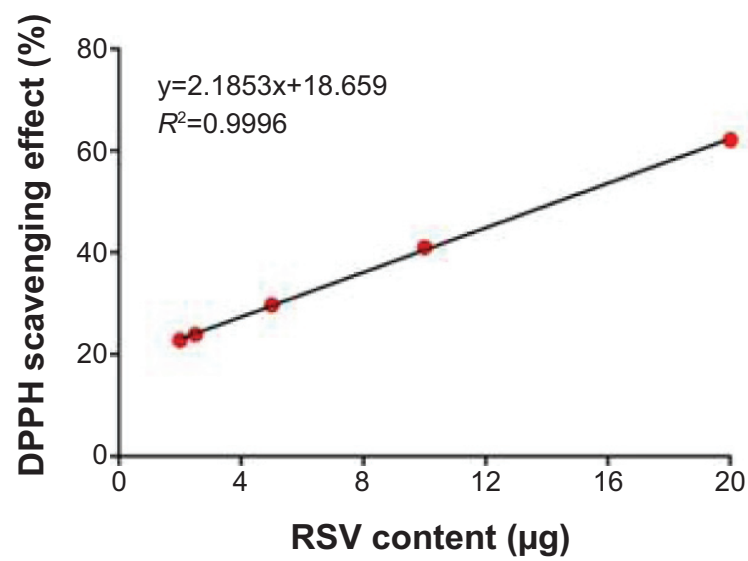

C

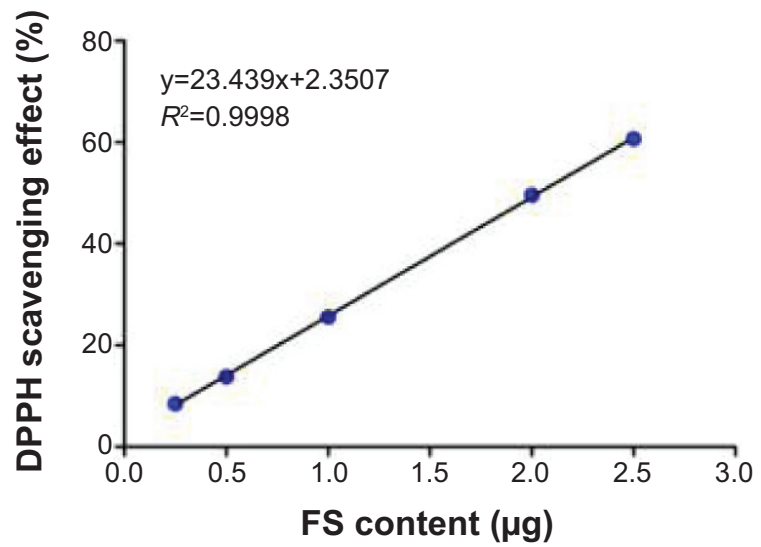

Figure S3 Antioxidant activity of (A) EGCG, (B) RSV, and (C) FS raw materials at various concentrations assessed by percentage inhibition of DPPH.

Abbreviations: EGCG, epigallocatechin-3-gallate; RSV, resveratrol; FS, fisetin; DPPH, 2,2-diphenyl-I-picrylhydrazyl.
A

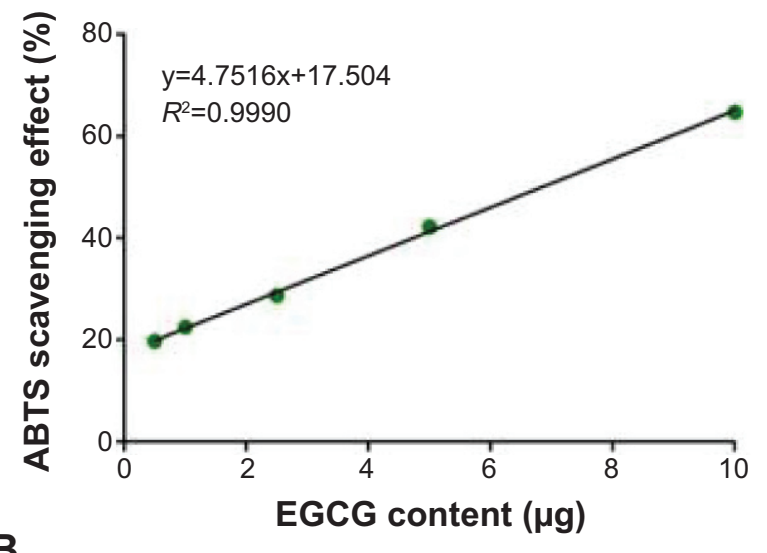

B
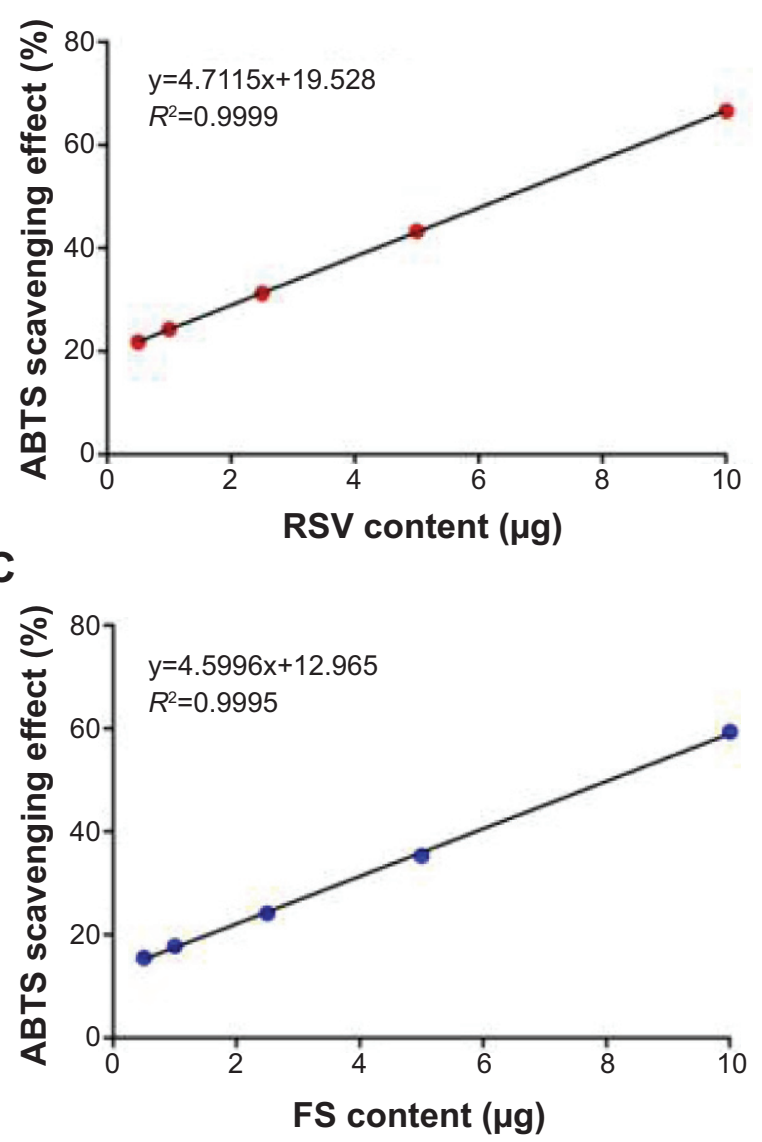

Figure S4 Antioxidant activity of (A) EGCG, (B) RSV, and (C) FS raw materials at various concentrations assessed by percentage inhibition of $A B T S$.

Abbreviations: EGCG, epigallocatechin-3-gallate; RSV, resveratrol; FS, fisetin; ABTS, 2,2'-azinobis(3-ethylbenzothiazoline-6-sulfonic acid) cation. 
A

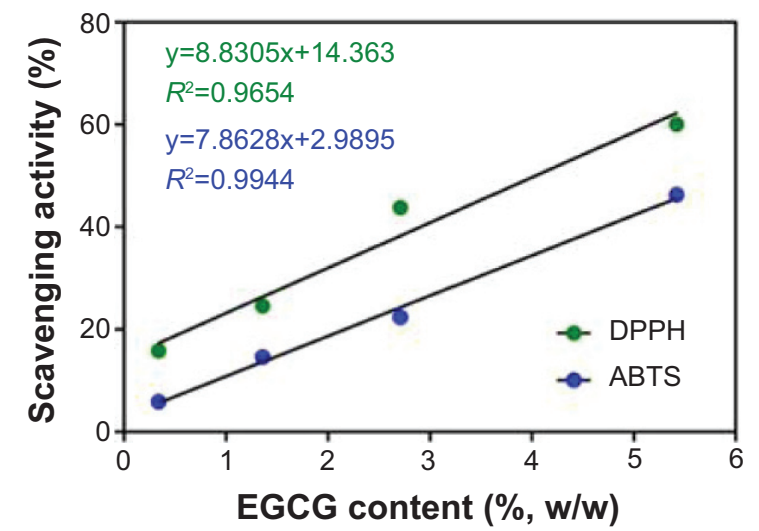

B

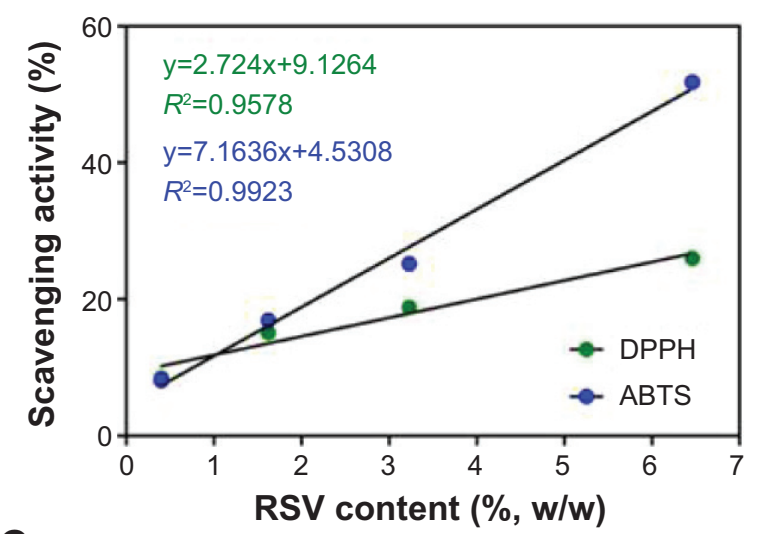

C

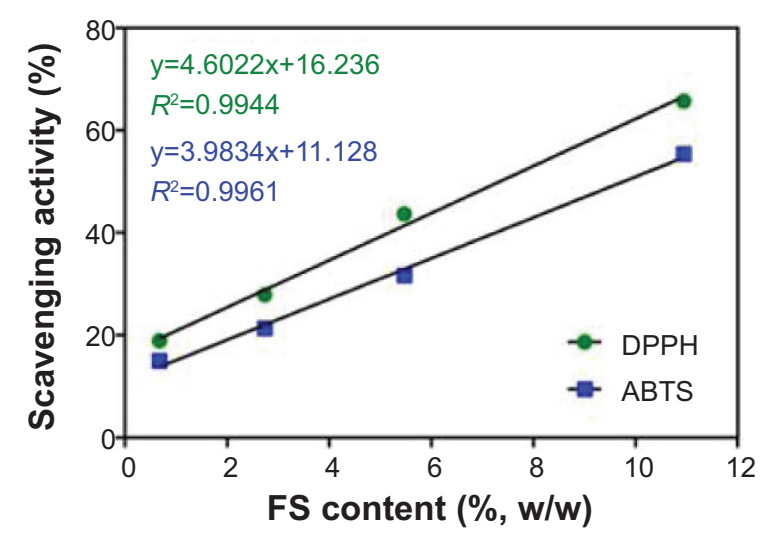

Figure S5 Correlation between radical-scavenging capacity assays (DPPH and ABTS) of EGCG-GNPs, RSV-GNPs, and FS-GNPs and (A) EGCG, (B) RSV, and (C) FS content $(\%, w / w)$.

Abbreviations: DPPH, 2,2-diphenyl-I-picrylhydrazyl; ABTS, 2,2'-azinobis (3-ethylbenzothiazoline-6-sulfonic acid) cation; EGCG, epigallocatechin-3-gallate; GNPs, gold nanoparticles; RSV, resveratrol; FS, fisetin.
A

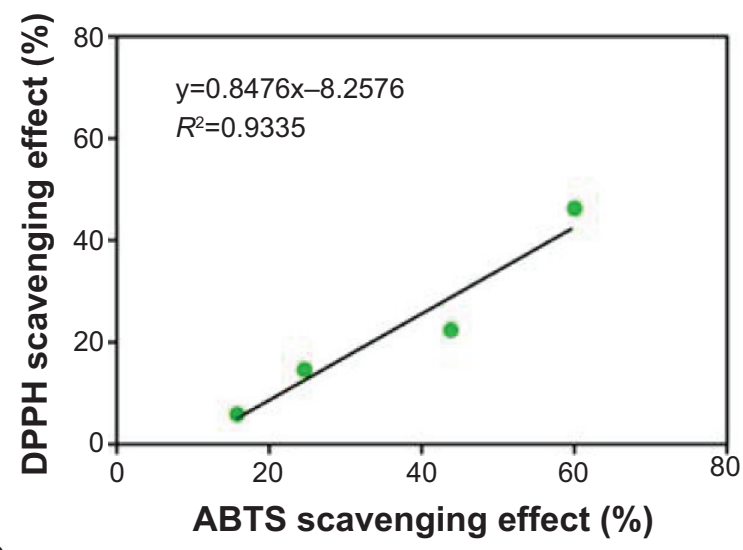

B

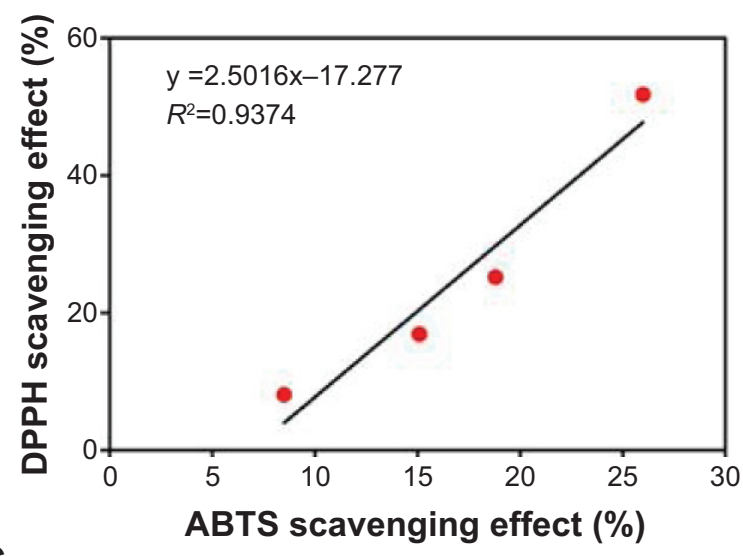

C

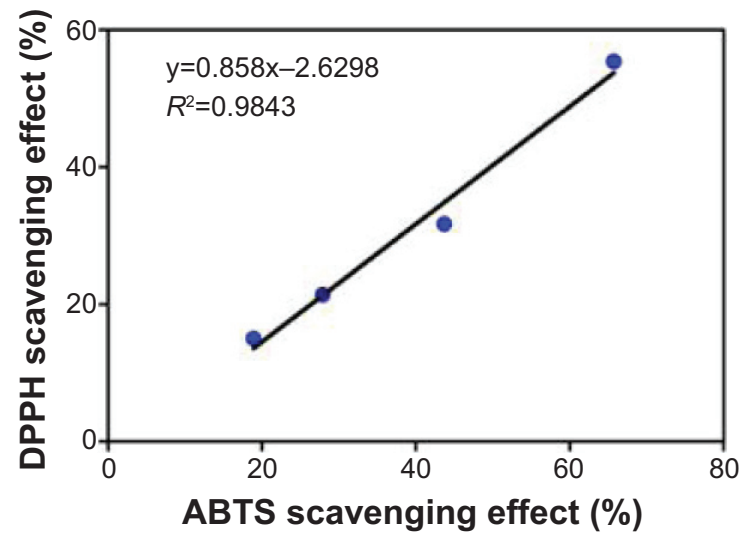

Figure S6 Correlation between DPPH and ABTS assays of (A) EGCG-GNPs, (B) RSV-GNPs, and (C) FS-GNPs.

Abbreviations: DPPH, 2,2-diphenyl-I-picrylhydrazyl; $\quad A B T S, \quad 2,2^{\prime}$-azinobis (3-ethylbenzothiazoline-6-sulfonic acid) cation; EGCG, epigallocatechin-3-gallate; GNPs, gold nanoparticles; RSV, resveratrol; FS, fisetin.
International Journal of Nanomedicine

\section{Publish your work in this journal}

The International Journal of Nanomedicine is an international, peerreviewed journal focusing on the application of nanotechnology in diagnostics, therapeutics, and drug delivery systems throughout the biomedical field. This journal is indexed on PubMed Central, MedLine, CAS, SciSearch ${ }^{\circledR}$, Current Contents ${ }^{\circledR} /$ Clinical Medicine,

\section{Dovepress}

Journal Citation Reports/Science Edition, EMBase, Scopus and the Elsevier Bibliographic databases. The manuscript management system is completely online and includes a very quick and fair peer-review system, which is all easy to use. Visit http://www.dovepress.com/ testimonials.php to read real quotes from published authors. 